
\title{
$\angle$ Research Square \\ Modeling and analysis of the friction in a nonlinear sliding-mode triboelectric energy harvester
}

Dongguo Tan

Hunan University

Jiaxi Zhou ( $\boldsymbol{\sigma}$ jxizhou@hnu.edu.cn )

Hunan University https://orcid.org/0000-0001-8348-6859

Kai Wang

Hunan University

Changqi Cai

Hunan University

Daolin Xu

Hunan University

\section{Research Article}

Keywords: Triboelectric energy harvester, Magnetic bistability, Macro-slip friction model, Coulomb friction model, Nonlinearity

Posted Date: April 13th, 2021

DOl: https://doi.org/10.21203/rs.3.rs-394015/v1

License: (c) (i) This work is licensed under a Creative Commons Attribution 4.0 International License.

Read Full License 


\title{
Modeling and analysis of the friction in a nonlinear sliding-mode triboelectric energy harvester
}

\author{
Dongguo Tan, Jiaxi Zhou*, Kai Wang*, Changqi Cai, Daolin Xu \\ College of Mechanical and Vehicle Engineering, Hunan University, Changsha 410082, PR China
}

\begin{abstract}
Friction plays a pivotal role in the sliding-mode triboelectric energy harvester (TEH), which not only enables the charge transfer between two dielectrics, but also influences the energy harvesting performance by affecting the dynamic response of the TEH. How to evaluate the effects of the friction on TEHs is important for optimizing TEHs in engineering practices. In order to analyze the effects of the friction on the dynamic response and evaluate the energy harvesting performance of TEHs, the paper models the friction of a devised nonlinear TEH based on the Coulomb friction model and the Macro-slip friction theory. The TEH equips a pair of magnets, rendering a switching between the bistability and the monostability by tuning the distance between two magnets. The dynamic model of the nonlinear TEH is established by the extended Hamilton principle. The effects of the friction in sliding-mode TEH are dissected in detail. The influences of parameters on both the mechanical and electrical responses are also systemically studied, to explore an optimal energy harvesting performance in the low-frequency range. This work provides a guideline for designing and accurately analyzing a sliding-mode TEH.
\end{abstract}

Keywords: Triboelectric energy harvester; Magnetic bistability; Macro-slip friction model; Coulomb friction model; Nonlinearity

\footnotetext{
${ }^{*}$ Corresponding author.

Address: College of Mechanical and Vehicle Engineering, Hunan University, Changsha 410082, PR China.

E-mail address: jxizhou@hnu.edu.cn (Jiaxi Zhou); Telephone number: +86 13975835883.

${ }^{*}$ Corresponding author.

Address: College of Mechanical and Vehicle Engineering, Hunan University, Changsha 410082, PR China.

E-mail address: wangkai@hnu.edu.cn (Kai Wang); Telephone number: +86 18011510813.
} 


\section{Introduction}

With the rapid growth of fifth-generation $(5 \mathrm{G})$ wireless communication in the world $[1,2]$, the application of tiny components and miniature sensor become widely. It is becoming an urgent topic among researchers and engineers that how to power the huge sensor network. Compared with traditional batteries, harvesting ambient energy (such as friction, mechanical vibration, water wave and human motion) has the limitless foreground, since its huge reserves and pollution-free property. Owing to the dependence between the dynamical response and the energy harvesting performance, the essential issue becomes how to design a novel configuration to enhance the efficiency of converting ambient energy to electrical energy.

Ordinarily, the possible mechanisms for harvesting ambient energy include electromagnetic mechanism $[3,4]$, piezoelectric mechanism $[5,6]$, pyroelectric mechanism $[7,8]$ and electrostatic mechanism $[9,10]$. The fundamental principle of the electromagnetic energy harvester can be boiled down to the law of electromagnetic induction. In terms of the piezoelectric energy harvester, the piezoelectric patches can harvest electric energy when they are extruded with mechanical strain [11]. It should be pointed out that the piezoelectric energy harvester possesses higher energy conversion efficiency at the high-frequency range. Unlike the electromagnetic and the piezoelectric energy harvester, the pyroelectric energy harvester achieves energy conversion by temperature fluctuation [12].

As an additional mechanism of energy harvesting, electrostatic induction occurs in two triboelectric materials when they have opposite polarity [9]. In this state, positive and negative charges are on the two different materials, respectively. When the two triboelectric materials slide each other, charges transfer from one triboelectric material to another one, causing an electric current. In 2012, Fan et al. proposed a triboelectric energy harvester (TEH) based on electrostatic induction [13]. After that, a large number of scholars have been attracted by the THEs due to easy manufacture and widespread applications [14]. In order to obtain higher harvesting efficiency and 
energy output, the vertical contact separation mode TEH was presented [15]. Moreover, a variety of different modes TEHs were proposed by using the same theory of electrostatic induction, for instance, the in-plane sliding-mode [16] and the single-electrode mode [17]. According to these proposed triboelectric mechanism, a huge number of structures have been devised to broaden the frequency bandwidth [18, $19]$ and improve the low-frequency vibration energy conversion efficiency [20, 21]. In addition, it was found that nonlinear energy harvesters have better performance of energy harvesting compared with the linear energy harvesters [22-25].

In order to obtain a great performance of energy harvesting, scholars often design nonlinear TEHs [26-30]. For example, Wang et al. [31] presented a nonlinear hybrid energy harvester with electromagnetic generator and triboelectric generator, which could harvest energy at high ultralow-frequency. Fu et al [32] proposed a TEH based on a three-degree-of-freedom vibro-impact oscillator, and it can harvest energy from low-frequency ambient vibration. Yang et al [33] investigated a magnetic levitation-based tristable hybrid energy harvester for scavenging energy from low-frequency structural vibration. Wang et al. [34] presented a tri-hybrid energy harvester that can generate higher output power at lower frequencies by interwell oscillations. All of these work indicated that nonlinear TEHs can improve the efficiency of converting energy from mechanical vibration to electrical energy by broadening the frequency bandwidth in low-frequency range.

Generally, the energy harvesting performance of TEHs is closely related to the structural vibration. In addition, friction plays a pivotal role in structural dynamics by affecting the dynamic response in TEHs. Originally, a linear damping was used to describe the dissipation energy caused by friction, but the nonlinear properties induced by friction are lost through this simplification. As a simple interface model, Coulomb friction model is often used to describe the friction relationship between two contact surfaces, which assumes that the contact points do not move with respect to each other unless the friction force exceeds a critical limit [35]. Later, Macro-slip friction model was presented based on the Coulomb friction model with considering contact stiffness [36]. Compared with Coulomb friction model, Macro-slip friction 
model is more accurate [37, 38]. In order to simplify the analysis for TEHs, the friction between the two dielectrics is often simplified. For instance, the friction is simplified as a linear viscous damping in Ref. [21]. Fortunately, Fu et al. [39] first investigated the friction in a sliding-mode TEH based on the Coulomb friction model, which pioneered the research about the effect of the friction on the performance of TEHs. However, the systematic researches about friction in TEHs are still sporadic, scarce and rudimentary, resulting in a difficulty to design and evaluate a TEH more accurately.

This paper proposes a sliding-mode TEH consisting of a novel bi-stable bird-shaped cantilever. The Macro-slip friction model is employed to describe the slip-stick behavior, and the modeling of the TEH is established by using Hamilton principle. The structural dynamics are achieved to evaluate the energy harvesting performance by resolving this model numerically. The main contribution of this paper is attempting to reveal the undelaying principle of slip-stick behavior between dielectric films in the sliding-mode TEHs, and thus to find out how the friction affect the energy harvesting efficiency of the TEHs.

This paper is organized as following. In Section 2, the TEH is designed and the working mechanism of the TEH is described. The modeling of the TEH system and the theory of Macro-slip friction model are also presented in this section. In Section 3, the dynamic behaviors of the TEH are investigated, and several factors impacting the dynamic behaviors, especially the friction model, are discussed. Based on the Macro-slip friction model, the electrical performances of the TEH are evaluated in Section 4. Finally, some conclusions are drawn in Section 5.

\section{Theoretical Modeling}

\subsection{Model and principle}

The conceptual model of the TEH is shown in Fig. 1. The mechanical structure of the TEH includes two parts, namely a bird-shaped cantilever and a pair of magnets. 
The left-hand end of the cantilever is clamped and a cube magnet (Magnet 1) is adhered to its right free end. Another magnet (Magnet 2) is fixed opposite to Magnet 1 , to repel the Magnet 1 when the cantilever is in horizontal position. The structure utilized to capture the vibration energy contains two dielectrics, two electrodes and two friction plates, as shown in Fig. 1b. One of the friction plates (Fiction plate 1) is fixed on a cantilever, forming a bird-shaped cantilever. Another friction plate (Fiction plate 2) is hinged at the base, and a normal force acts on this plate to enable friction between Dielectric 1 and Dielectric 2 . The tangential contact stiffness $k_{\mathrm{d}}$ is taken into account. For the Coulomb friction model, $k_{\mathrm{d}}$ is infinite, while it is a constant for the Macro-slip model. When the cantilever is oscillated by the base excitation, reciprocating sliding appears between Friction plate 1 and Friction plate 2.

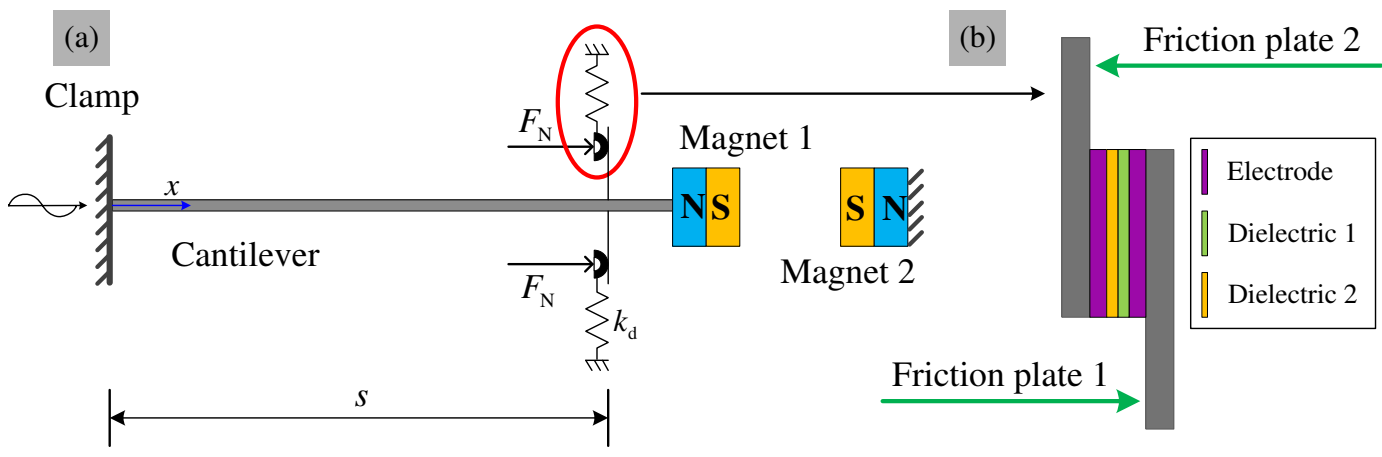

Fig. 1 Conceptual model of the TEH

As shown in Fig. 1b, the dielectric and the electrode are attached to the friction plates. The operating principle of TEH [9] is given in Fig. 2. When the energy harvester is in the initial state, the contact area between Friction plate 1 and Friction plate 2 is depicted in Fig. 2a. In this state, Dielectric 1 and Dielectric 2 fully overlap each other. Owing to the different electron attracting capabilities, charges transfer from Dielectric 2 to Dielectric 1, and thus Dielectric 1 gains electrons and Dielectric 2 loses electrons. As a consequence, the surfaces of Dielectric 1 and Dielectric 2 are negatively and positively charged, respectively. 


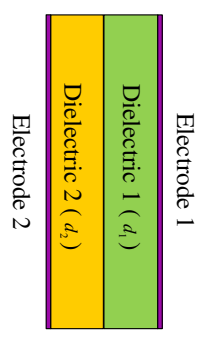

(a)

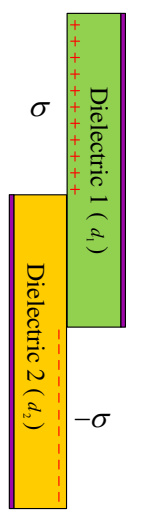

(b)

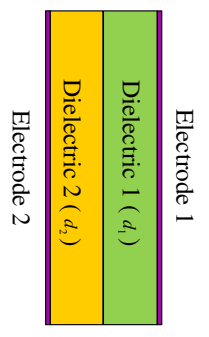

(c)

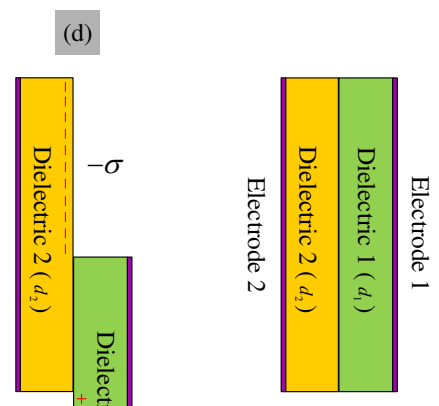

(e)

Fig. 2 The operating principle of TEH

When an excitation is applied on the nonlinear TEH, Friction plate 1, which is connected with the cantilever, repels Dielectric 2 and Electrode 2 far away from the initial position of the nonlinear TEH. As illustrated in Fig. 2b, Dielectric 1 deviates from the initial position with an upward distance. An electric potential difference between Dielectric 1 and Dielectric 2, induced by the instability of the charge distribution, is generated and results in a flow of electrons from Electrode 1 to Electrode 2. When Dielectric 1 moves up to the maximum distance, it follows the oscillation of the cantilever and begins to slide down. Under this circumstance, the flow of electrons is from Electrode 2 to Electrode 1 until they are perfect overlapped, as shown in Fig. 2c. After that, Dielectric 1 continues to slide down and reaches the maximum distance in the downward direction, as shown in Fig. 2d. After that, the Dielectric 2 will move upward and reaches the initial position.

\subsection{Modeling of the mechanical system}

The extended Hamilton principle is used to derive the equation of motion of the nonlinear TEH under external excitations. The general variational equation of the TEH can be given as

$$
\delta \int_{t_{1}}^{t_{2}}(T-U) \mathrm{d} t+\int_{t_{1}}^{t_{2}} \delta W \mathrm{~d} t=0
$$


here, $\delta$ is the infinitesimal variation, $T=T_{\mathrm{b}}+T_{\mathrm{m} 1}$ where $T_{\mathrm{b}}$ and $T_{\mathrm{m} 1}$ are the kinetic energy of cantilever and the kinetic energy of Magnet 1, respectively. $U$ is the potential energy that can be written as $U=U_{\mathrm{b}}+U_{\mathrm{m}}$, where $U_{\mathrm{b}}$ and $U_{\mathrm{m}}$ denote the elastic potential energy of cantilever and the potential energy in the magnetic field, respectively. $W$ is the work done by external forces, including the friction force. $t_{1}$ is the start time and $t_{2}$ is the end time.

Since the mass of Friction plate 1 is negligible compared to the cantilever beam, the kinetic energy $T_{\mathrm{b}}$ can be given by

$$
T_{\mathrm{b}}=\frac{1}{2} \int_{0}^{L_{\mathrm{c}}} \rho_{\mathrm{c}} b_{\mathrm{c}} h_{\mathrm{c}}\left[\frac{\partial w(x, t)}{\partial t}+\frac{\mathrm{d} u(t)}{\mathrm{d} t}\right]^{2} \mathrm{~d} x
$$

where $w(x, t)$ denotes the transverse displacement, $L_{\mathrm{c}}$ is the length of the cantilever beam, $\rho_{\mathrm{c}}$ is the density of the cantilever.$b_{\mathrm{c}}$ and $h_{\mathrm{c}}$ are the width and height of the cantilever beam's cross-sectional area, respectively. The base displacement is defined as $u(t)$.

The kinetic energy of Magnet 1 can be evaluated by

$$
T_{\mathrm{m} 1}=\frac{1}{2} m\left[\left.\frac{\partial w(x, t)}{\partial t}\right|_{x=L_{\mathrm{c}}}+\frac{\mathrm{d} u(t)}{\mathrm{d} t}\right]^{2}+\frac{1}{2} I_{\mathrm{t}}\left[\left.\frac{\partial w(x, t)}{\partial x \partial t}\right|_{x=L_{\mathrm{c}}}\right]^{2}
$$

where the mass of Magnet 1 is $m$, and $I_{\mathrm{t}}$ is the rotational inertia of the mass of the tip magnet.

The elastic potential energy $U_{\mathrm{b}}$ is written as

$$
U_{\mathrm{b}}=\frac{1}{2} \int_{0}^{L_{\mathrm{e}}} E I\left(\frac{\partial^{2} w(x, t)}{\partial x^{2}}\right)^{2} \mathrm{~d} x
$$

where $E I$ is the bending stiffness of the cantilever beam.

In actual situation, the locations of the two magnets in the motion states are presented in Fig. 3 [40, 41]. The tip magnet (Magnet 1) is subjected to the magnetic force of the fixed magnet (Magnet 2). 


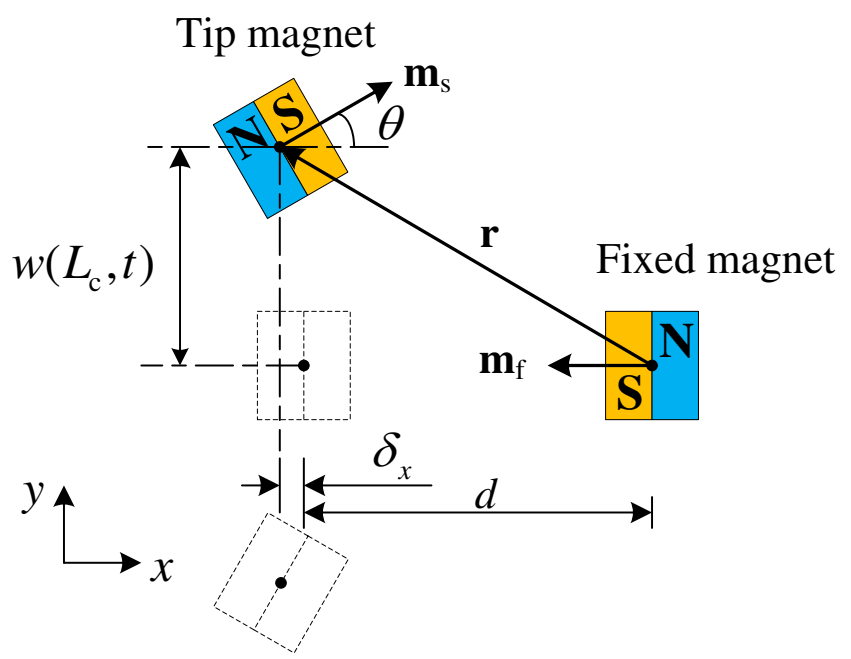

Fig. 3 Geometries configuration of the two magnets

The elastic potential energy generated by the magnetic field can be expressed as

$$
U_{\mathrm{m}}=-\mathbf{B} \cdot \mathbf{m}_{\mathrm{s}}
$$

where $\mathbf{m}_{\mathrm{s}}$ and $\mathbf{B}$ are the magnetic moment vector of the tip magnet and the magnetic field generated by the fixed magnet at the location of tip magnet, respectively. The magnetic field $\mathbf{B}$ can be evaluated by

$$
\mathbf{B}=-\frac{\mu_{0}}{4 \pi} \nabla \frac{\mathbf{m}_{\mathrm{f}} \cdot \mathbf{r}}{\|\mathbf{r}\|_{2}^{3}}
$$

where $\mu_{0}$ is the vacuum permeability, $\mathbf{m}_{\mathrm{f}}$ denotes the magnetic moment vector of the fixed magnet, $\nabla$ and \|\|$_{2}$ represent the vector gradient operator and L2-norm, respectively. $\mathbf{r}$ is the vector from the fixed magnet to that of the tip magnet and the corresponding expression is

$$
\mathbf{r}=-\left(d+\delta_{x}\right) \hat{x}+w\left(L_{\mathrm{c}}, t\right) \hat{y}
$$

where $d$ shows the distance between the center of the tip magnet in base status and the center of the fixed magnet, $\hat{x}$ and $\hat{y}$ represent the unit vectors along the $x$-axis and the $y$-axis, respectively. The longitudinal displacement of the tip magnet $\delta_{x}$ can 
be expressed as $\delta_{x}=\frac{1}{2} \int_{0}^{L_{c}}\left[\frac{\partial w(x, t)}{\partial x}\right]^{2} \mathrm{~d} x$.

In theory, gradient functions [42] are defined as follows

$$
\begin{gathered}
\nabla \frac{1}{r^{n}}=\left(\begin{array}{l}
\partial / \partial x \\
\partial / \partial y \\
\partial / \partial z
\end{array}\right) \frac{1}{r^{n}}=-\frac{n}{r^{n+1}}\left(\begin{array}{l}
x / r \\
y / r \\
z / r
\end{array}\right)=-\frac{n \mathbf{r}}{r^{n+1}} \\
\nabla\left(\mathbf{v}_{1} \cdot \mathbf{r}\right)=\left(\begin{array}{c}
\partial / \partial x \\
\partial / \partial y \\
\partial / \partial z
\end{array}\right)\left(\mathbf{v}_{1} \cdot \mathbf{r}\right)=\left(\begin{array}{l}
\partial / \partial x\left(x_{1} x+y_{1} y+z_{1} z\right) \\
\partial / \partial y\left(x_{1} x+y_{1} y+z_{1} z\right) \\
\partial / \partial z\left(x_{1} x+y_{1} y+z_{1} z\right)
\end{array}\right)=\left(\begin{array}{l}
x_{1} \\
y_{1} \\
z_{1}
\end{array}\right)=\mathbf{v}_{1}
\end{gathered}
$$

The magnetic field Eq. (6) can be derived by Eq. (8) and Eq. (9), substituting Eq. (6) into Eq. (5) yield

$$
U_{\mathrm{m}}=\frac{\mu_{0}}{4 \pi}\left[\frac{1}{r^{3}}\left(\mathbf{m}_{\mathrm{s}} \cdot \mathbf{m}_{\mathrm{f}}\right)-\frac{3}{r^{5}}\left(\mathbf{m}_{\mathrm{s}} \cdot \mathbf{r}\right)\left(\mathbf{m}_{\mathrm{f}} \cdot \mathbf{r}\right)\right]
$$

where $r$ is the L2-norm of the vectors $\mathbf{r}$ and it can be written as $r=\left[(d+\delta x)^{2}+w^{2}\left(L_{\mathrm{c}}, t\right)\right]^{1 / 2}$.

In the magnetic field, $\mathbf{m}_{\mathrm{s}}$ and $\mathbf{m}_{\mathrm{f}}$ can be given by

$$
\begin{aligned}
& \mathbf{m}_{\mathrm{s}}=M_{\mathrm{s}} V_{\mathrm{s}}(\cos \theta \bar{x}+\sin \theta \bar{y}) \\
& \mathbf{m}_{\mathrm{f}}=-M_{\mathrm{f}} V_{\mathrm{f}} \hat{x}
\end{aligned}
$$

where $M_{\mathrm{s}}$ and $M_{\mathrm{f}}$ are the magnitude of the magnetization of the tip magnet and the fixed magnet, respectively. And $M_{\mathrm{s}}$ can be described as $M_{\mathrm{s}}=B_{\mathrm{r}} / \mu_{0}$ by using the residual flux density $B_{\mathrm{r}} . V_{\mathrm{s}}$ and $V_{\mathrm{f}}$ are the tip magnet's volume and the fixed magnet's volume, respectively. $\theta$ is the slope of the cantilever at the tip magnet.

Substituting Eq. (7) and Eq. (11) into Eq. (10), one can get the expressions of the elastic potential energy as

$$
U_{\mathrm{m}}=\frac{\mu_{0} M_{\mathrm{s}} V_{\mathrm{s}} M_{\mathrm{f}} V_{\mathrm{f}}}{4 \pi r^{3}}\left[-\cos \theta-\frac{3}{r^{2}}\left[-\left(d+\delta_{x}\right)^{2} \cos \theta+w\left(L_{\mathrm{c}}, t\right)\left(d+\delta_{x}\right) \sin \theta\right]\right]
$$

Assuming the first linear mode is dominant of the cantilever beam, the transverse displacement $w(x, t)$ can be approximated as 


$$
w(x, t)=\phi(x) q(t)
$$

where $q(t)$ represents the temporal modal coordinate, the mode shape function $\phi(x)$ can be given by

$$
\phi(x)=C[\cos \beta x-\cosh \beta x+\xi(\sin \beta x-\sinh \beta x)]
$$

where $C$ is determined from the corresponding orthonormality conditions, $\beta$ and $\xi$ can be derived from the eigensystem. The boundary conditions of a cantilever beam is used as

$$
\begin{aligned}
& \left.w(x, t)\right|_{x=0}=0 \\
& \left.\frac{\partial w(x, t)}{\partial x}\right|_{x=0}=0 \\
& \left.\frac{\partial^{2} w(x, t)}{\partial x}\right|_{x=L_{\mathrm{c}}}=0 \\
& \left.\frac{\partial^{3} w(x, t)}{\partial x}\right|_{x=L_{\mathrm{c}}}=0
\end{aligned}
$$

Substituting Eq. (13) into Eq. (2) to (5), kinetic energies of the cantilever beam and the tip magnet can be derived, potential energies of the cantilever beam and the magnetic field also can be obtained, and they are expressed as

$$
\begin{aligned}
& T_{\mathrm{b}}=\frac{1}{2} \int_{0}^{L_{\mathrm{c}}} \rho_{\mathrm{c}} b_{\mathrm{c}} h_{\mathrm{c}}\left(\phi^{2} \dot{q}^{2}+2 \phi \dot{q} \dot{u}+\dot{u}^{2}\right) \mathrm{d} x \\
& T_{\mathrm{m} 1}=\frac{1}{2} m\left[\phi^{2}\left(L_{\mathrm{c}}\right) \dot{q}^{2}+2 \phi\left(L_{\mathrm{c}}\right) \dot{q} \dot{u}+\dot{u}^{2}\right]+\frac{1}{2} I_{\mathrm{t}}\left[\phi^{\prime}\left(L_{\mathrm{c}}\right)\right]^{2} \dot{q}^{2} \\
& U_{\mathrm{b}}=\frac{1}{2} \int_{0}^{L_{\mathrm{c}}} E I\left(\phi^{\prime \prime}\right)^{2} q^{2} \mathrm{~d} x \\
& U_{\mathrm{m}}=\frac{\mu_{0} M_{\mathrm{s}} V_{\mathrm{s}} M_{\mathrm{f}} V_{\mathrm{f}}}{4 \pi r^{3}}\left[-\cos \theta-\frac{3}{r^{2}}\left[-\left(d+\delta_{x}\right)^{2} \cos \theta+\phi\left(L_{\mathrm{c}}\right) q\left(d+\delta_{x}\right) \sin \theta\right]\right]
\end{aligned}
$$

where 


$$
\begin{aligned}
& \delta_{x}=\frac{1}{2} \int_{0}^{L_{\mathrm{c}}}\left(\phi^{\prime}\right)^{2} q^{2} \mathrm{~d} x \\
& r=\left[(d+\delta x)^{2}+\left[q \phi\left(L_{\mathrm{c}}\right)\right]^{2}\right]^{1 / 2} \\
& \cos \theta=1-\frac{1}{2} q^{2}\left[\phi^{\prime}\left(L_{\mathrm{c}}\right)\right]^{2} \\
& \sin \theta=q \phi^{\prime}\left(L_{\mathrm{c}}\right)
\end{aligned}
$$

The Lagrange function for the mechanical system can be written as

$$
L=T_{\mathrm{b}}+T_{\mathrm{m} 1}-U_{\mathrm{b}}-U_{\mathrm{m}}
$$

The dynamical equation of the mechanical system can be evaluated by

$$
\frac{\mathrm{d}}{\mathrm{d} t}\left(\frac{\partial L}{\partial \dot{q}}\right)-\frac{\partial L}{\partial q}+F_{\mathrm{f}} \phi(s)=0
$$

According to Eq. (13) and Eqs. (16) - (19), Eq. (19) can be derived as

$$
\ddot{q}+2 \zeta \omega \dot{q}+\omega^{2} q+\frac{F_{\mathrm{m}}}{M}=\frac{\alpha}{M} \ddot{u}-\frac{F_{\mathrm{f}} \phi(s)}{M}
$$

where $\zeta$ is the structural damping ratio, $\omega$ is the undamped natural frequency of the cantilever beam without the tip magnet's mass, $F_{\mathrm{m}}$ is the magnetic force and it can obtained by $\partial U_{\mathrm{m}} / \partial q, \quad \ddot{u}=A \sin \left(\omega_{\mathrm{u}} t\right)$ is the base excitation, $A$ is the acceleration amplitude, $\omega_{u}=2 \pi f$ and $f$ is the frequency. $F_{\mathrm{f}}$ is the friction force. The expression for $\alpha$ and the first modal mass $M$ are given by

$$
\begin{aligned}
& \alpha=-\left[\rho_{\mathrm{c}} b_{\mathrm{c}} h_{\mathrm{c}} \int_{0}^{L_{\mathrm{c}}} \phi(x) \mathrm{d} x+m_{\mathrm{s}} \phi\left(L_{\mathrm{c}}\right)\right] \\
& M=\left[\rho_{\mathrm{c}} b_{\mathrm{c}} h_{\mathrm{c}} \int_{0}^{L_{\mathrm{c}}} \phi^{2}(x) \mathrm{d} x+m_{\mathrm{s}} \phi^{2}\left(L_{\mathrm{c}}\right)+I_{\mathrm{t}}\left[\phi^{\prime}\left(L_{\mathrm{c}}\right)\right]^{2}\right]
\end{aligned}
$$

\subsection{Marco-slip friction model}

The mathematical relationship between nonlinear friction force and relative displacement on the contact surface is described in the friction model, and this friction model can be divided in to Macro-slip model and Micro-slip model [43-45]. In this article, the Macro-slip model is employed owing to the almost same accuracy and less calculation cost. As a counterpart, the Coulomb friction model is also utilized to 
describe the friction.

Assuming that the base excitation is harmonic, the relative displacement of the contact point on the friction plate can be given by

$$
w=X \cos (\varpi \varsigma+\varphi)=X \cos \vartheta
$$

where $X$ is the amplitude, $\varpi$ and $\varphi$ are the vibration angular frequency and initial phase, respectively.

As according to the concerned Marco-slip model, the non-linear friction force, $F_{\mathrm{f} \_\mathrm{M}}$, can be expressed as

$$
F_{\mathrm{f}_{-} \mathrm{M}}=\left\{\begin{array}{lll}
k_{\mathrm{d}}\left(w-w_{\mathrm{r}}\right) & \text { when } & k_{\mathrm{d}}\left|w-w_{\mathrm{r}}\right| \leq \mu F_{\mathrm{N}} \\
\mu F_{\mathrm{N}} \operatorname{sgn}\left(\dot{w}_{\mathrm{r}}\right) & \text { when } & k_{\mathrm{d}}\left|w-w_{\mathrm{r}}\right| \geq \mu F_{\mathrm{N}}
\end{array}\right.
$$

where $w_{\mathrm{r}}$ is the displacement of the friction plate, and $k_{\mathrm{d}}$ is the tangential contact stiffness of the friction plates. Fig. 4 shows the relationship between the response and the friction force. The system are in stick state and slip state during the $A B$ and $B C$ sections, respectively. The velocity of the friction plate $\dot{w}_{\mathrm{r}}$ can be expressed as

$$
\dot{w}_{\mathrm{r}}=\left\{\begin{array}{lll}
0 & \text { when } & 0 \leq \vartheta \leq \vartheta^{*} \\
\dot{w} & \text { when } & \vartheta^{*} \leq \vartheta \leq \pi
\end{array}\right.
$$

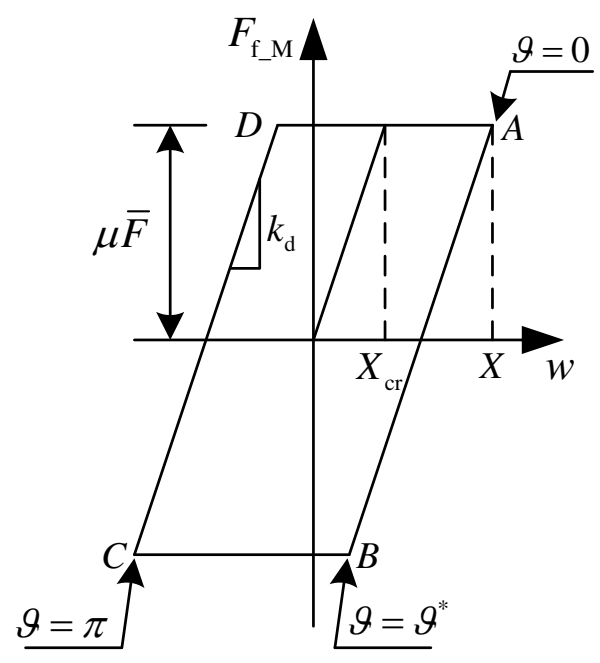

Fig. 4 Frictional damping loop, friction force versus the response

The displacement $w_{\mathrm{r}}$ can be obtained by integrating Eq. (24) 


$$
w_{\mathrm{r}}=\left\{\begin{array}{lll}
c_{1} & \text { when } & 0 \leq \vartheta \leq \vartheta^{*} \\
w+c_{2} & \text { when } & \vartheta^{*} \leq \vartheta \leq \pi
\end{array}\right.
$$

In the stick state (as denoted by symbol $A B$ in Fig. 4), the friction force is equal to zero when $w$ is equal to $w_{\mathrm{r}}$. Thus, $c_{1}=X-\mu F_{\mathrm{N}} / k_{\mathrm{d}}$. During the slip state (as denoted by symbol $B C$ ), the difference between $w$ and $w_{\mathrm{r}}$ is the constant $c_{2}$. When $w_{\mathrm{r}}$ is at point $B$, one can find that $c_{2}$ is the horizontal distance between point $B$ and the middle point of line $A B$ in Fig. 4. Therefore, $c_{2}=\mu F_{\mathrm{N}} / k_{\mathrm{d}}$. From Eq. (23), the friction force can be given by

$$
\begin{gathered}
F_{\mathrm{f}_{-} \mathrm{M}}=k_{\mathrm{d}}\left(w-w_{\mathrm{r}}\right)=k_{\mathrm{d}}(w-X)+\mu F_{\mathrm{N}} \quad \text { when } \quad 0 \leq \vartheta \leq \vartheta^{*} \\
F_{\mathrm{f} \_\mathrm{M}}=\mu F_{\mathrm{N}} \operatorname{sgn}\left(\dot{w}_{\mathrm{r}}\right)=\mu F_{\mathrm{N}} \operatorname{sgn}(\dot{w})=-\mu F_{\mathrm{N}} \quad \text { when } \quad \vartheta^{*} \leq \vartheta \leq \pi
\end{gathered}
$$

when $\vartheta=\vartheta^{*}$, one can yield

$$
\vartheta^{*}=\arccos \left[1-2 \mu F_{\mathrm{N}} /\left(k_{\mathrm{d}} X\right)\right]
$$

Since the response is assumed to be a simple harmonic form, the response cycle can be divided into two symmetric half cycles, i.e., the half cycle $A B C$ is equal to the other half cycle $C D A$ in Fig. 4. Thus, one can find

$$
F_{\mathrm{f} \_\mathrm{M}}(\vartheta+\pi)=-F_{\mathrm{f} \_\mathrm{M}}(\vartheta) \quad 0 \leq \vartheta \leq \pi
$$

Therefore, the nonlinear friction force corresponding to the sliding state between the contact surfaces of the friction plates can be expressed as

$$
F_{\mathrm{f}}=\left\{\begin{array}{l}
F-2 \mu F_{\mathrm{N}}\left[1-\left(1+\frac{X \cos \vartheta-X}{2 X_{\mathrm{cr}}}\right)\right] \quad 0 \leq \vartheta \leq \vartheta^{*} \\
-\mu F_{\mathrm{N}} \quad \vartheta^{*} \leq \vartheta \leq \pi \\
-F+2 \mu F_{\mathrm{N}}\left[1-\left(1-\frac{X \cos \vartheta+X}{2 X_{\mathrm{cr}}}\right)\right] \quad \pi \leq \vartheta \leq \pi+\vartheta^{*} \\
\mu F_{\mathrm{N}} \quad \pi+\vartheta^{*} \leq \vartheta \leq 2 \pi
\end{array}\right.
$$

where $F=\mu F_{\mathrm{N}}, \vartheta^{*}=\arccos \left(1-2 X_{\mathrm{cr}} / X\right)$ and $X_{\mathrm{cr}}$ can be given by $\mu F_{\mathrm{N}} / k_{\mathrm{d}}$.

When the system enters into a steady-state, periodically changed friction force on the contact surfaces is presented, and the friction force can be represented by the sum 
of the equivalent restoring and damping forces, as given by

$$
F_{\mathrm{f}}=k_{\mathrm{e}} w(s, t)+c_{\mathrm{e}} \dot{w}(s, t)
$$

where $k_{\mathrm{e}}$ and $c_{\mathrm{e}}$ are the equivalent stiffness and equivalent damping coefficients, respectively, which can be given by

$$
\begin{aligned}
& k_{\mathrm{e}}= \begin{cases}\frac{k_{\mathrm{d}}}{\pi}\left(\vartheta^{*}-\frac{1}{2} \sin 2 \vartheta^{*}\right) & \left(X>X_{\mathrm{cr}}\right) \\
k_{\mathrm{d}} & \left(X \leq X_{\mathrm{cr}}\right)\end{cases} \\
& c_{\mathrm{e}}= \begin{cases}\frac{4 \mu F_{\mathrm{N}}}{\pi \omega X} \frac{X-X_{\mathrm{cr}}}{\pi} & \left(X>X_{\mathrm{cr}}\right) \\
0 & \left(X \leq X_{\mathrm{cr}}\right)\end{cases}
\end{aligned}
$$

Substituting Eqs. (13), (31) and (32) into Eq. (20) yields the dynamical equation with the Marco-slip friction model

$$
\ddot{q}+\left[2 \zeta \omega+\frac{c_{\mathrm{e}} \phi^{2}(s)}{M}\right] \dot{q}+\left[\omega^{2}+\frac{k_{\mathrm{e}} \phi^{2}(s)}{M}\right] q+\frac{F_{\mathrm{m}}}{M}=\frac{\alpha}{M} \ddot{u}
$$

The equivalent stiffness and damping coefficients are shown as Fig. 5. Because $X_{\mathrm{cr}}$ is in direct proportion to the normal force, $X / X_{\mathrm{cr}}$ rises with the decrease in the normal force. As the normal force decreases, the equivalent stiffness diminishes and the damping coefficients increases first and then decreases. When the normal force closes to infinity, $k_{\mathrm{e}}$ and $c_{\mathrm{e}}$ approach to $k_{\mathrm{d}}$ and zero, respectively.
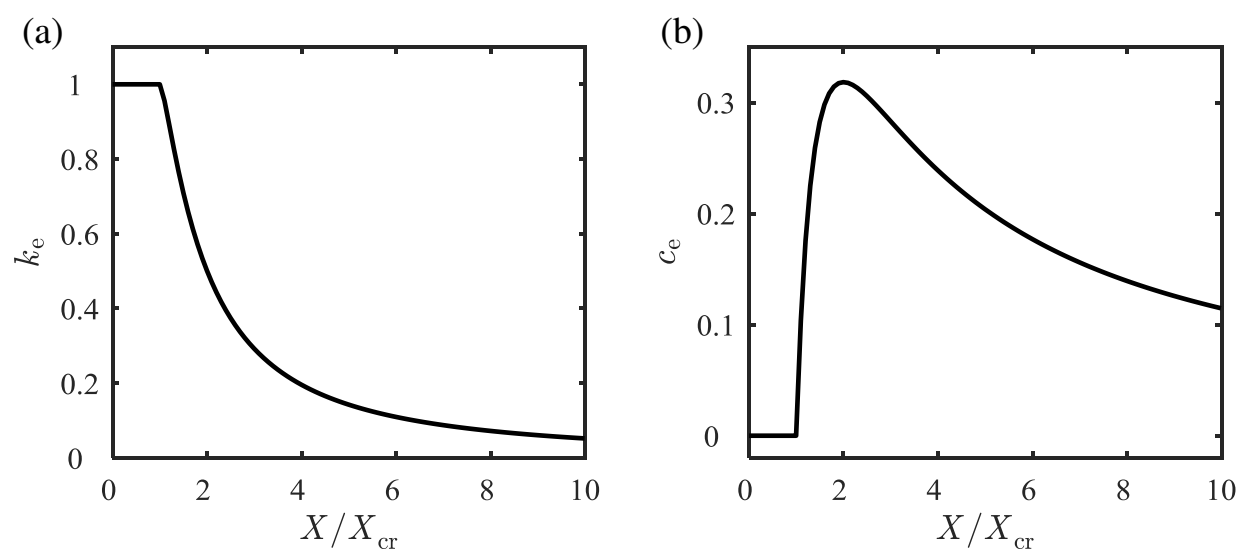

Fig. 5 The variation of (a) equivalent stiffness, (b) equivalent damping coefficients

Note that the Macro-slip model degrade to the Coulomb friction model when the tangential stiffness $k_{\mathrm{d}}$ is infinite. According to Coulomb's law of friction, the 
kinetic friction force on the slider can be given as

$$
F_{\mathrm{f}_{-} \mathrm{C}}=\mu F_{\mathrm{N}} \operatorname{sgn}(\dot{w})=c_{\mathrm{e}_{-} \mathrm{C}} \dot{w}
$$

where $\mu$ and $F_{\mathrm{N}}$ are the coefficient of friction and normal force on contact surfaces of friction plates, respectively. $\operatorname{sgn}(\cdot)$ is the signum function, $c_{\mathrm{e}_{-} \mathrm{C}}$ is the equivalent damping of Coulomb friction model.

In the Eq. (34), the term $\operatorname{sgn}(\dot{w})$ is a periodic square wave function which can be expanded into a Fourier series. Including the fundamental harmonic term only, Eq. (34) can be rewritten as

$$
F_{\mathrm{f}_{-} \mathrm{C}}=\mu F_{\mathrm{N}} \operatorname{sgn}(\dot{w}) \approx-\frac{4 \mu F_{\mathrm{N}}}{\pi} \sin (\varpi \varsigma+\varphi)
$$

Substituting Eq. (35) into Eq. (34), one can get the equivalent damping of Coulomb friction model

$$
c_{\mathrm{e}_{-} \mathrm{C}}=\frac{4 \mu F_{\mathrm{N}}}{\pi \varpi X}
$$

\section{Dynamic behaviors of the TEH}

In this section, the equations of motion are solved by the Runge-Kutta method. The system parameters employed for numerical simulations are tabulated in Table 1.

Table 1 The parameters employed for numerical simulations

\begin{tabular}{lll}
\hline Parameter & symbol & value \\
\hline Length of the cantilever beam & $L_{\mathrm{c}}$ & $200 \mathrm{~mm}$ \\
Width of the cross-sectional area of the beam & $b_{\mathrm{c}}$ & $10 \mathrm{~mm}$ \\
Height of the cross-sectional area of the beam & $h_{\mathrm{c}}$ & $1 \mathrm{~mm}$ \\
Material density of the beam & $\rho_{\mathrm{c}}$ & $7800 \mathrm{~kg} / \mathrm{m}^{3}$ \\
Young's modulus of cantilever & $E$ & $210 \mathrm{Gpa}$ \\
Poisson's ratio & $v$ & 0.3 \\
Damping ratio & $\zeta$ & 0.0038 \\
\hline
\end{tabular}


Mass of Magnet 1 or Magnet 2

The vacuum permeability

Residual flux density of magnet

Volume of Magnet 1

Volume of Magnet 2

Friction coefficient

$\begin{array}{ll}m & 12.8 \mathrm{~g} \\ \mu_{0} & 4 \pi \times 10^{-7} \\ B_{\mathrm{r}} & 1.48 \\ V_{\mathrm{s}} & 8 \times 8 \times 8 \mathrm{~mm}^{3} \\ V_{\mathrm{f}} & 8 \times 8 \times 8 \mathrm{~mm}^{3} \\ \mu & 0.2\end{array}$

\subsection{Bistability of the TEH}

In the $\mathrm{TEH}$, only the restoring force of the cantilever beam and the magnetic force between the tip and fixed magnets are considered. The restoring force is merely affected by the deflection of the beam, and the magnetic force is influenced by the distance between the magnets, as illustrated in Fig. 6a. In effect, the strain energy of the cantilever beam and the magnetic potential energy depend on the restoring force and the magnetic force, respectively. Thus, the total force is composed of the restoring force and the magnetic force.

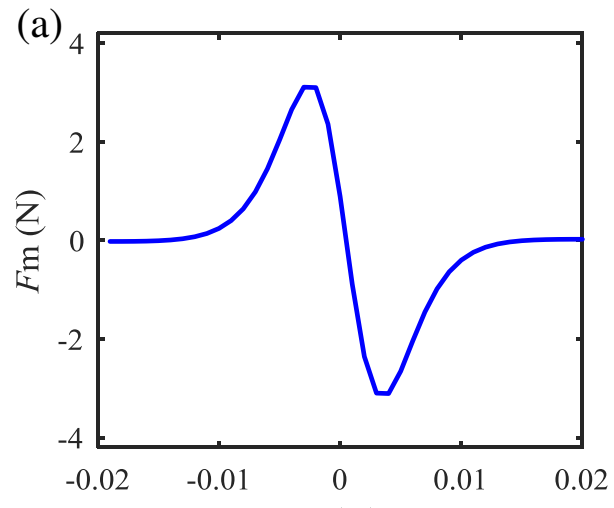

(c)
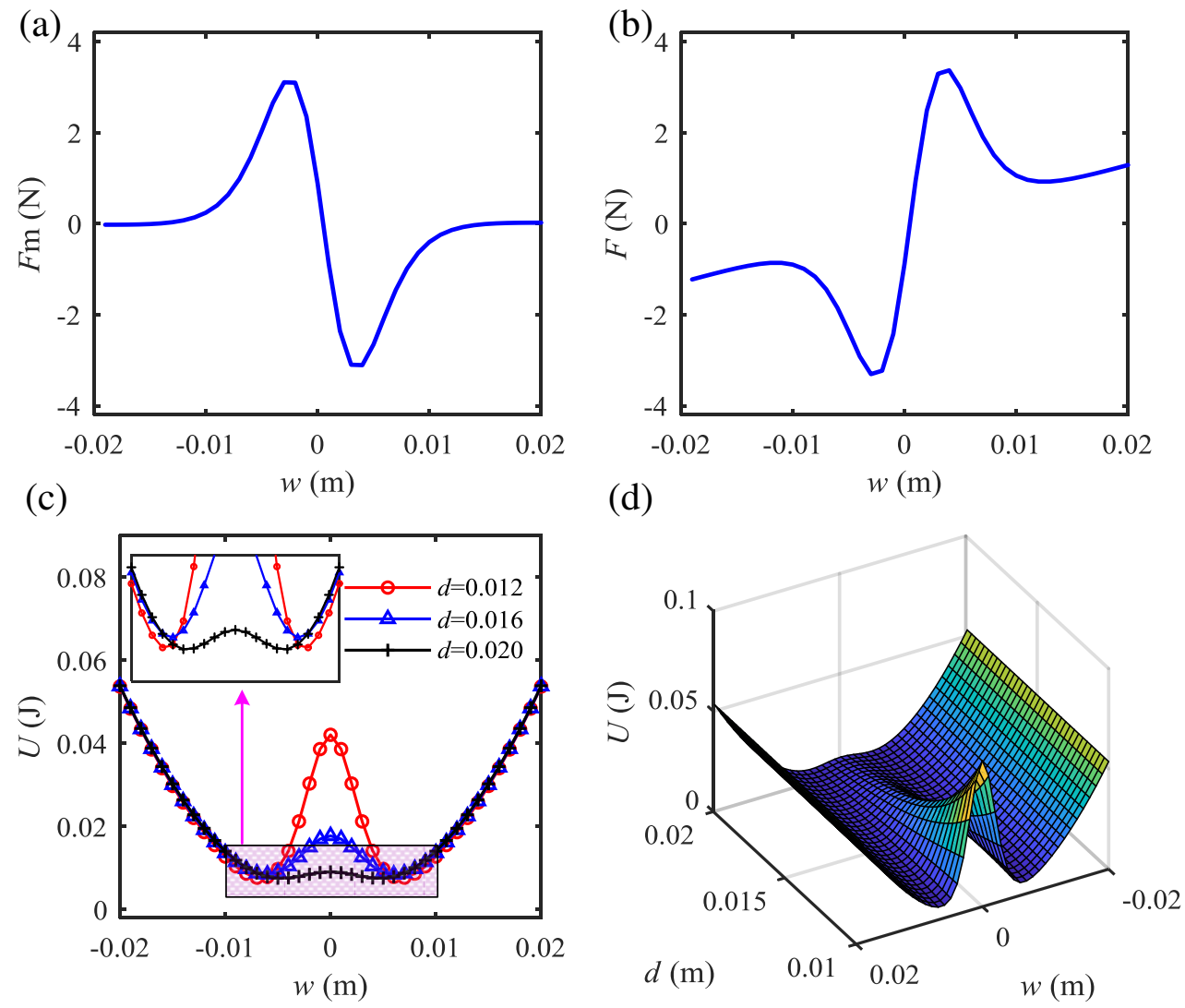
Fig. 6 The variation of the force with the tip displacement, (a) the magnetic force, (b) the total force; the relationship between the total potential energy and the tip displacement, (c) for the different horizontal distances $d$, (d) a 3D surface plot under horizontal distances sweeps.

Fig. $6 \mathrm{c}$ shows the relationship between the total potential energy of the TEH without friction and the tip displacement of the cantilever beam for different horizontal distances between the magnets $(d=0.012 \mathrm{~m}, 0.016 \mathrm{~m}$ and $0.02 \mathrm{~m})$. When the horizontal distance is small, the tip magnet and the fixed magnet are close, and the system exhibits significant bistability. In order to obtain a more intuitive variation of the total potential energy against the horizontal distance, a 3D surface plot is presented in Fig. 6d. Clearly, the bistability of the system gets weaker as the horizontal distance increases. If the magnets are far enough apart, the magnetic field has little effect on the tip magnet, and the system becomes monostable.

\subsection{Effect of the friction}

The TEH harvests energy primarily through electron transfer of triboelectrification, and friction is the main factor influencing triboelectrification. Meanwhile, friction causes energy dissipation by means of heat. Thus, friction is very complex in the nonlinear TEH, and current researches on the TEH are sorely lack. In this section, Marco-slip friction model is applied to describe the slip-stick behavior of the system, which is also compared with the Coulomb friction model. In addition, the performance of the TEH is evaluated by investigating the effects of the system parameters on the dynamic characteristics.

\subsubsection{Normal force}

Based on the Coulomb model and Macro-slip model, the effect of normal force on the vibration amplitude of the slider (Friction plate 1) in the TEH is reported in Fig. 7. Fig. 7a and Fig. $7 \mathrm{~b}$ show the vibration response of the Friction plate 1 changing against the excitation frequency for different normal force (such as I $F_{\mathrm{N}}=0.1 \mathrm{~N}$, II 
$F_{\mathrm{N}}=0.2 \mathrm{~N}$ and III $F_{\mathrm{N}}=0.3 \mathrm{~N}$ ) by using the Coulomb model and Macro-slip model, respectively.

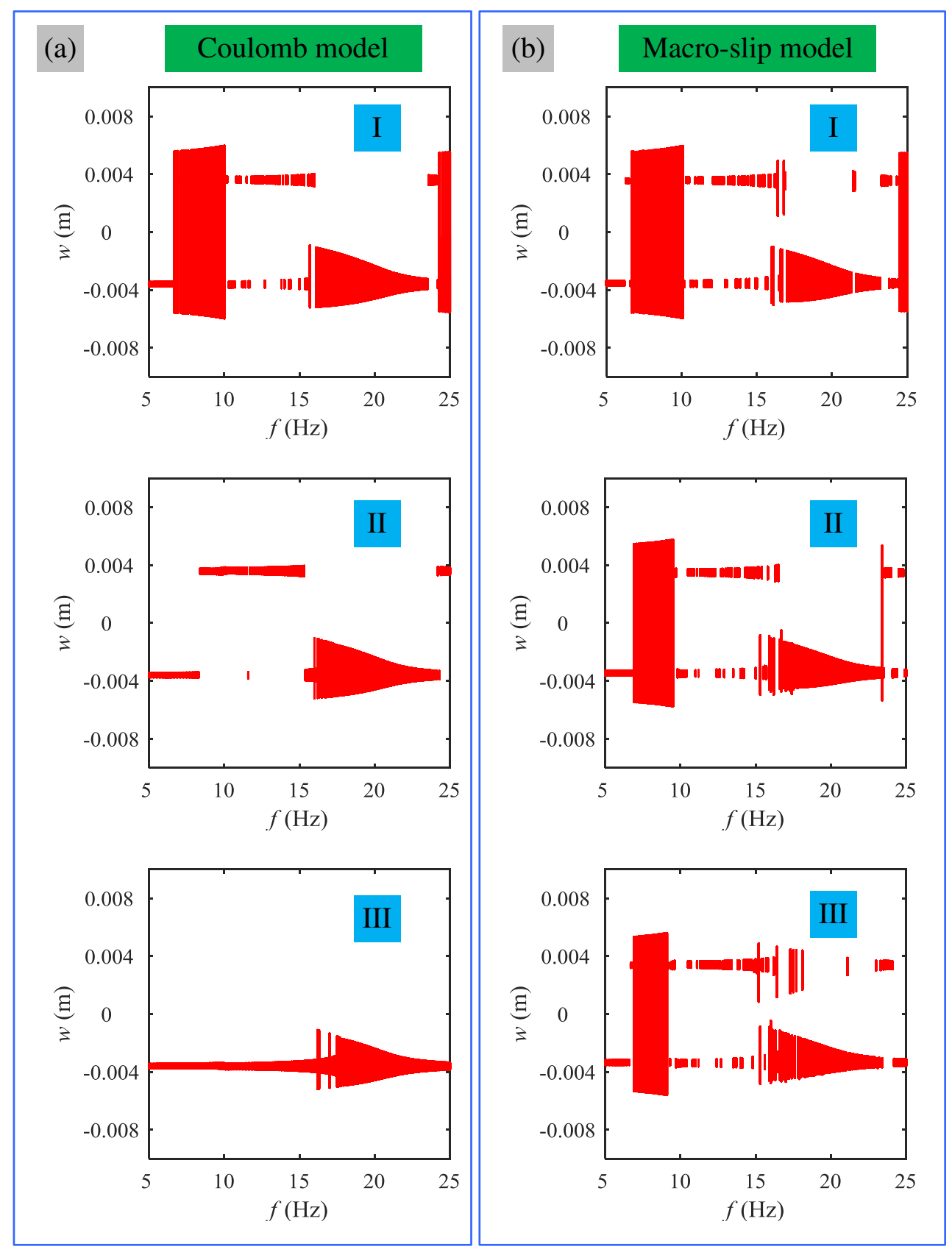

Fig.7 The effect of the normal force on the peak-peak value of dynamical response when I $F_{\mathrm{N}}=0.1 \mathrm{~N}$, II $\quad F_{\mathrm{N}}=0.2 \mathrm{~N}$, III $\quad F_{\mathrm{N}}=0.3 \mathrm{~N}, A=0.3 \mathrm{~g}, d=0.016 \mathrm{~m}, u=0.2$, and $k_{\mathrm{d}}=30$.

As depicted in Fig. 7a-I and Fig. 7b-I, interwell oscillations coexists with intrawell oscillations when the normal force is equal to $0.1 \mathrm{~N}$. The interwell oscillations occur in low-frequency region, because the friction force is small and the 
TEH is in slip state with large amplitudes. In addition, the motion pattern in Fig. 7a-I is same as that presented in Fig. 7b-I. The reason is that the TEH crosses the critical condition easier in low-frequency region and the slip state is dominant in Macro-slip model case when the normal force is tiny (e.g. $\left.F_{\mathrm{N}}=0.1 \mathrm{~N}\right)$. Thus, the Macro-slip model is equivalent to the Coulomb model as the normal force is small enough.

When the normal force increases from $0.1 \mathrm{~N}$ to $0.2 \mathrm{~N}$, however, the motion pattern of the TEH obtained by the Coulomb model is different from that acquired by the Macro-slip model. In terms of results calculated by the Coulomb model, as illustrated in Fig. 7a-II, the motion pattern of the TEH manifests in intrawell oscillations. That is, the TEH oscillates in one of the potential wells with a small amplitude. The reason is that the friction force is so large that the restoring force cannot offset the friction force and the magnetic force simultaneously. However, as reported in Fig. 7b-II, the TEH oscillates between two potential wells in some special low-frequency ranges, resulting in interwell oscillations with large amplitude when the Macro-slip model is utilized to calculate the dynamic responses of the TEH. Actually, such a difference can be attributed to that the friction force calculated by the Macro-slip model is smaller than that by the Coulomb model under the same normal force. More interestingly, the TEH is in slip state in low-frequency range, but this phenomenon cannot be revealed by the Coulomb model.

When the normal force continues to increase to $0.3 \mathrm{~N}$, the responses of the TEH obtained by the Coulomb model and the Macro-slip model are shown in Fig 7a-III and Fig 7b-III, respectively. From Fig 7a-III, it can be observed that the TEH cannot cross the potential energy barrier and undergoes intrawell oscillations, when the Coulomb model is utilized. However, the large-amplitude interwell oscillations appear in low-frequency range, when the Macro-slip model is employed, as shown in Fig 7b-III. Moreover, it is different from Fig 7b-II that the band of interwell oscillations decreases, and the interwell oscillations disappears at high frequency. It is because that the sliders of the TEH are in the stick state in a broader frequency range as the normal force increases. 
Generally, the large-amplitude interwell oscillations are expected for high-efficiency energy harvesting. Thus, the normal force corresponding to the occurrence of interwell oscillations is defined as critical normal force. For the Macro-slip friction model, the tangential contact stiffness has significant impact on the critical normal force, as shown in Fig. 8. Since the Coulomb friction model does not take into account the tangential contact stiffness, the critical normal force is invariable. Nevertheless, the Macro-slip friction model is different from Coulomb friction model. When the tangential contact stiffness is small, the Macro-slip friction model is approximately equal to Coulomb friction model. With the increases of the tangential contact stiffness, the critical normal force increases first and then decreases, as shown in Fig. 8. When tangential contact stiffness is sufficient large, the Macro-slip friction model is equivalent to Coulomb friction model again.

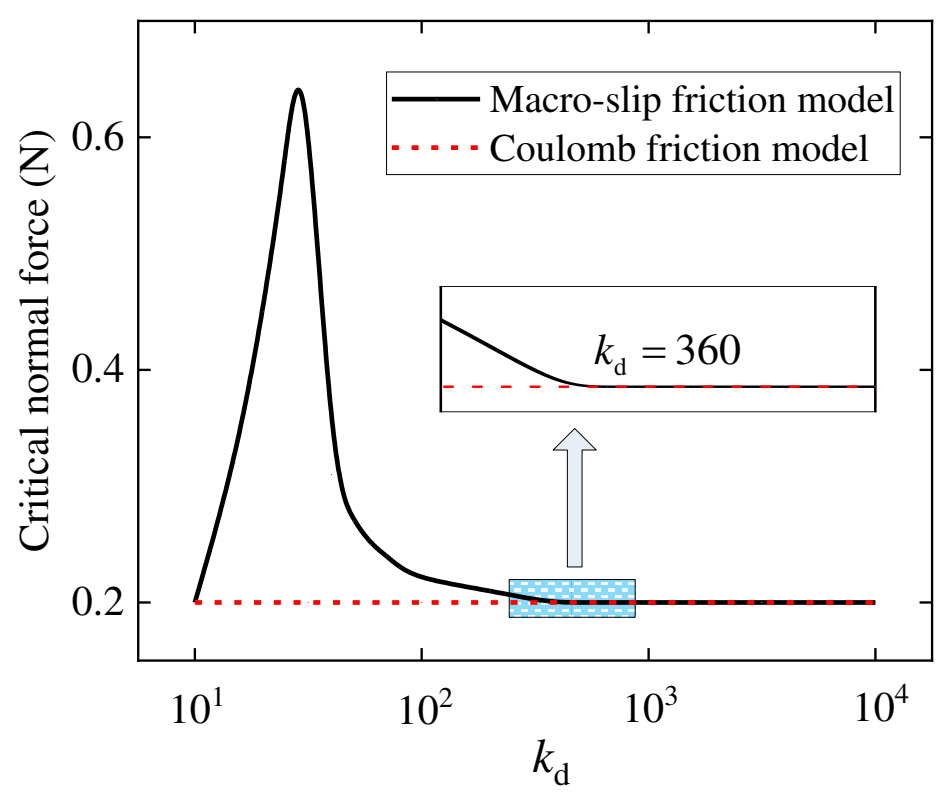

Fig.8 The critical normal force with different tangential contact stiffness.

As a consequence, Macro-slip model is equivalent to Coulomb model when the normal force is sufficient large or quite small. Moreover, a broader frequency band for interwell oscillations in low-frequency range can be achieved by using small normal forces. Hence, a small normal force is conducive to harvesting energy in the TEH. 


\subsubsection{Friction coefficient}

Fig. 9 shows the vibration amplitudes (peak-peak value) of Friction plate 1 under frequency sweeps with different friction coefficients (I $\mu=0.1$, II $\mu=0.2$, III $\mu=0.3$ ). The results shown in Fig. 9a and Fig. 9b are obtained based on the theory of Coulomb model and Macro-slip model, respectively.

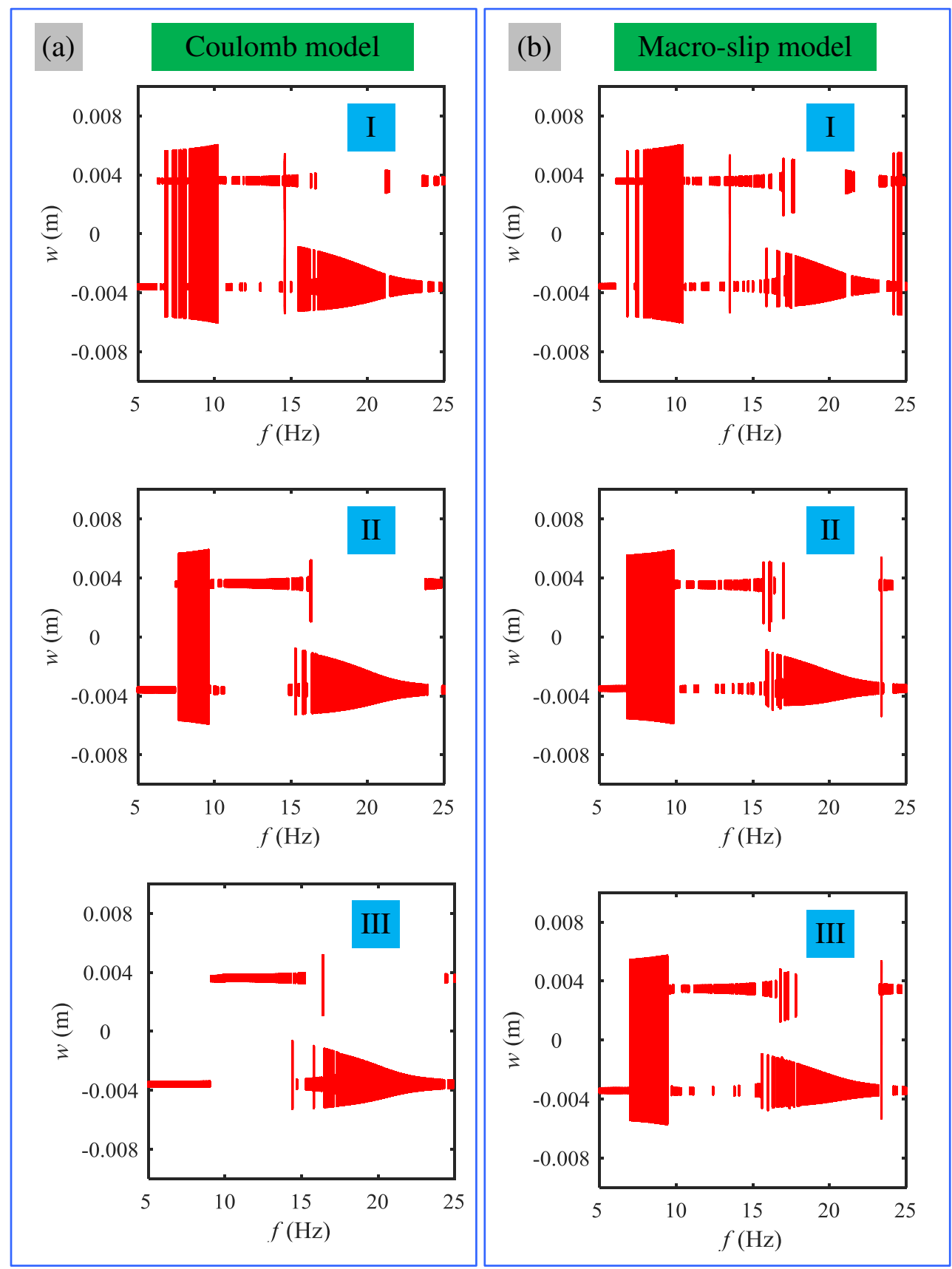

Fig.9 The influence of the friction coefficient on the peak-peak value of vibration amplitudes when I $\mu=0.1$, II $\mu=0.2$, III $\mu=0.3, A=0.3 \mathrm{~g}, d=0.016 \mathrm{~m}, F_{\mathrm{N}}=0.15 \mathrm{~N}$, and $k_{\mathrm{d}}=30$. 
As reported in Fig. 9a-I and Fig. 9b-I, the motion pattern of the TEH obtained by the Coulomb friction model is almost identical with that obtained by the Macro-slip friction model. When the friction coefficient is small, slip is easy to occur, according to Eq. (23). In such a case, the Macro-slip friction model is equivalent to the Coulomb friction model. It also can be seen that the TEH oscillates between two potential wells in low-frequency range, leading to large-amplitude responses which are beneficial to convert the vibration energy to electrical energy.

With the increase in the friction coefficient, as shown in Fig. 9a-II and Fig. 9b-II, the frequency band of interwell oscillations obtained by both the Coulomb friction model and the Macro-slip friction model get narrow. In addition, for the Coulomb friction model, the interwell oscillations in low-frequency range disappears as the friction coefficient increases from 0.2 to 0.3, as delineated in Fig. 9a-III. However, for the Macro-slip friction model, the frequency band of interwell oscillations is still can be observed and the bandwidth gets narrow, when the friction coefficient is increased to 0.3 .

Consequently, the Coulomb friction model would lead to incorrect predictions, when the normal force and friction coefficients are in a certain range, owing to the neglect of tangential contact stiffness. The following dynamic analysis and electrical feature of the TEH are conducted by employing the Macro-slip model.

\subsection{Effect of the distance between magnets}

As delineated in Fig. 6, the static characteristics of the nonlinear TEH are related to the distance between two magnets. Therefore, the distance between the two magnets plays a pivotal role on the dynamic response of the nonlinear $\mathrm{TEH}$, and thus impact the energy harvesting performance.

Fig. 10 shows the vibration responses of the Friction plate 1 change with the excitation frequency for different horizontal distance $d$ between the tip and fixed magnets by using the Macro-slip friction model. Clearly, when the distance is equal to 0.012 , the TEH is a typical bistable configuration with a high energy barrier. The 
magnetic force and the friction force are greater than the restoring force of cantilever beam, and the TEH cannot cross the potential energy barrier. Hence, the vibrations only exist in one side potential well and the motion of the tip magnet is intrawell oscillation, as shown in Fig. 10a. In addition, as $d$ increases, the magnetic force decreases. The TEH crosses the potential energy barrier from one potential well to another at some frequencies, but the motion of the tip magnet mainly is dominated by intrawell oscillations, as depicted in Fig. 10b. When $d$ continues to increase, the TEH jumps out of the potential energy wells in low-frequency range, and thus interwell oscillations coexists with intrawell oscillations in Fig. 10c. When the tip magnet is far apart from the fixed magnet, the structure of the TEH changes from bistability into monostabillity, due to a fact that the magnetic force is weak and the restoring force plays a dominant role, as shown in Fig. 10d.

Consequently, strong bistability can be fulfilled and a broad frequency band for interwell oscillations at low frequency can be achieved by selecting proper distance $d$ between the tip and fixed magnets, as shown in Fig. 10c. In such as case, the TEH has the excellent ability of energy harvesting from interwell oscillations over a broader frequency band. 

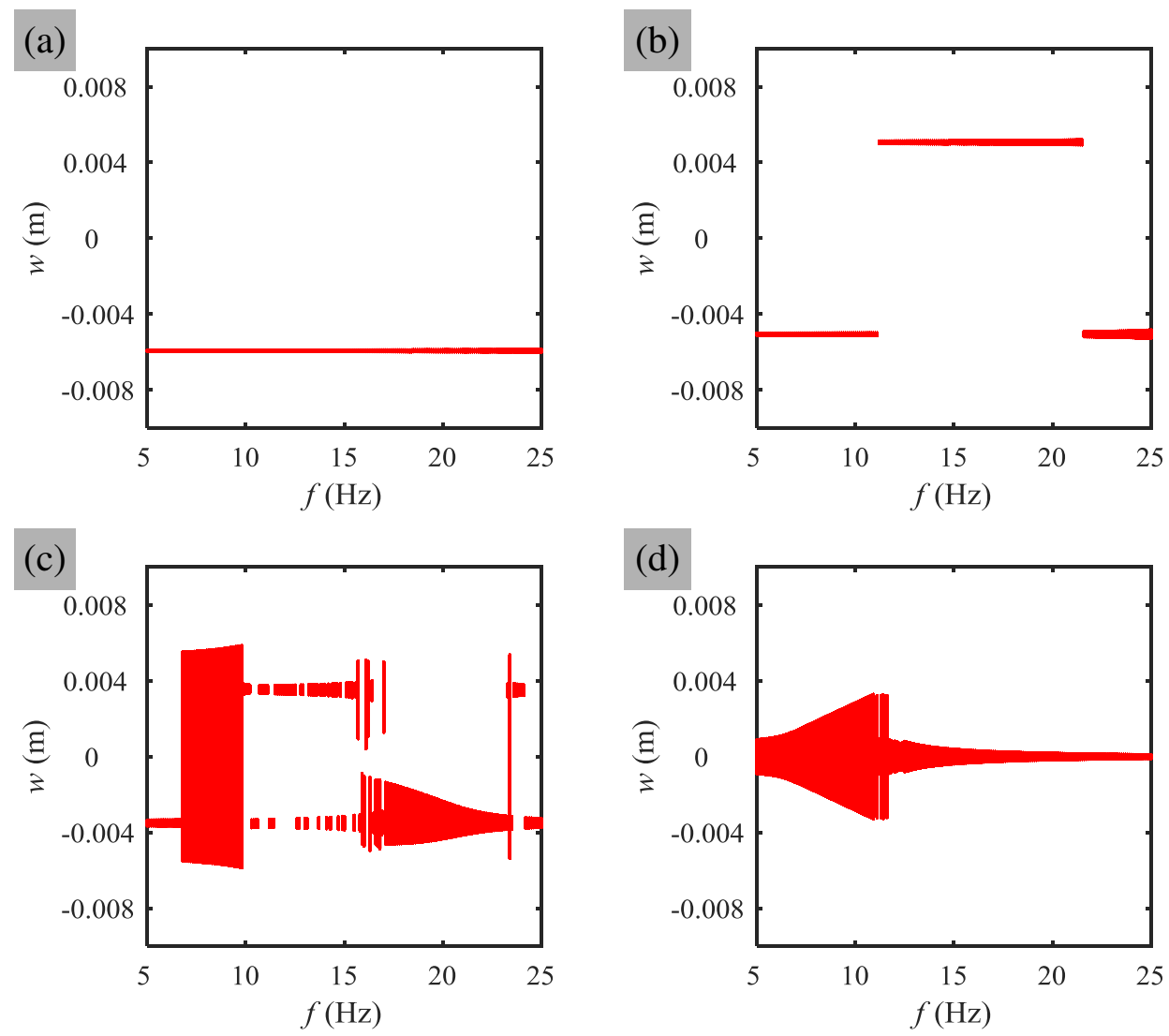

Fig. 10 Tip vibration responses of Friction plate 1 (peak-peak) under frequency sweeps with the horizontal distance $d$ between the tip magnet and the fixed magnet, (a) $d=0.012 \mathrm{~m}$, (b) $d=0.014 \mathrm{~m}$, (c) $d=0.016 \mathrm{~m},(\mathrm{~d}) d=0.018 \mathrm{~m} ; A=0.3 \mathrm{~g}, \quad F_{\mathrm{N}}=0.15 \mathrm{~N}, \quad k_{\mathrm{d}}=30$.

\subsection{Effect of the base excitation}

The level of base excitation has a significant contribution towards dynamic response of this proposed TEH. Fig. 11 shows the vibration amplitudes of friction plate under frequency sweeps with the acceleration amplitude of the base excitation through using the Macro-slip friction model. As shown in Fig. 11a, the TEH cannot cross the potential energy barrier since the acceleration amplitude is tiny (i.e., $A=0.1$ g), thus, the vibrations only exist in one side potential well. With the increase in the base excitation amplitude from $0.1 \mathrm{~g}$ to $0.2 \mathrm{~g}$, the motion of the tip magnet can cross the potential energy barrier at some frequency ranges, as displayed in Fig 11b. When the base excitations are increased to $A=0.3 \mathrm{~g}$ and $A=0.4 \mathrm{~g}$, the responses are reported 
in Fig. 11c and Fig. 11d, respectively. It can be observed that interwell oscillations coexist with intrawell oscillations, and the frequency band of interwell oscillations increases with the increase of the excitation. Therefore, high-level base excitation is beneficial to broaden the frequency band of interwell oscillations, and thus enhance the energy harvesting performance.
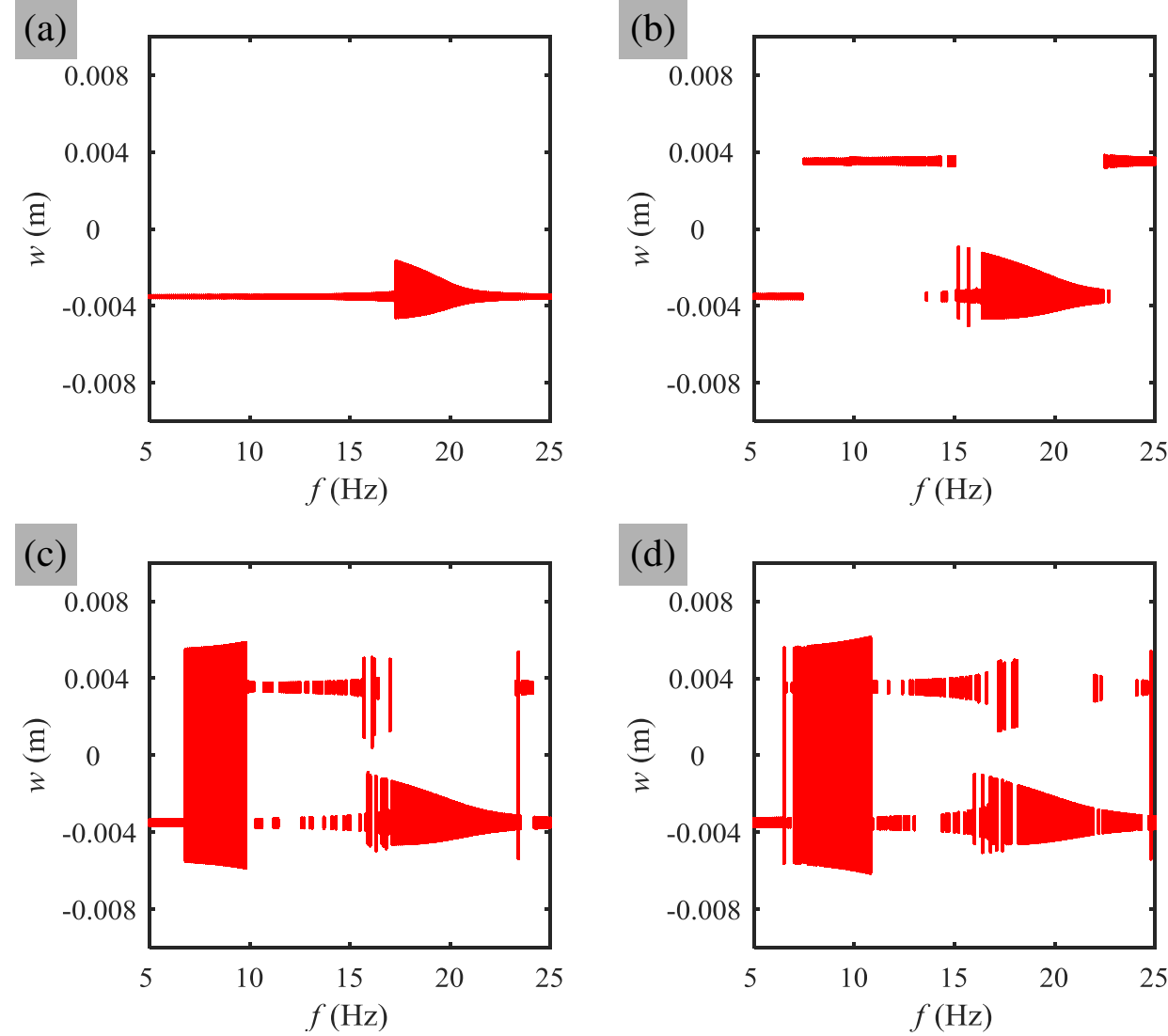

Fig. 11 Amplitudes of tip vibration (peak-peak) under frequency sweeps with the acceleration amplitude of the base excitation, (a) $A=0.1 \mathrm{~g}$, (b) $A=0.2 \mathrm{~g}$, (c) $A=0.3 \mathrm{~g}$, (d) $A=0.4 \mathrm{~g} ; d=0.016 \mathrm{~m}$, $F_{\mathrm{N}}=0.15 \mathrm{~N}, k_{\mathrm{d}}=30$.

\subsection{Influence of nonlinearity}

The system dynamics can be sensitively influenced by the excitation frequency and the horizontal distance between tip magnet and fixed magnet, and thus it is imperative to study the bifurcation of the responses about the excitation frequency and the horizontal distance. Therefore, the influences of the nonlinearity on the dynamical 
response of the TEH are carried out in this section based on the Macro-slip model.

Fig. 12a shows the bifurcation diagrams of the vibration amplitudes of the friction plate with respect to the excitation frequency, when the acceleration excitation amplitude is $A=1 \mathrm{~g}$ and the horizontal distance is $d=0.016 \mathrm{~m}$. As depicted in Fig. 12a, the motion is periodic when the excitation frequency is small. The responses of TEH turn into aperiodic/chaotic motion with the increase of the excitation frequency. Nonetheless, as the excitation frequency continues to increase, the system enters a periodic motion again. Fig. 12b shows the bifurcation diagrams of the responses of the TEH for the horizontal distance between magnets when $A=1 \mathrm{~g}$ and $f=8 \mathrm{~Hz}$. It can be observed that all the vibrations are periodic as the horizontal distance increases.
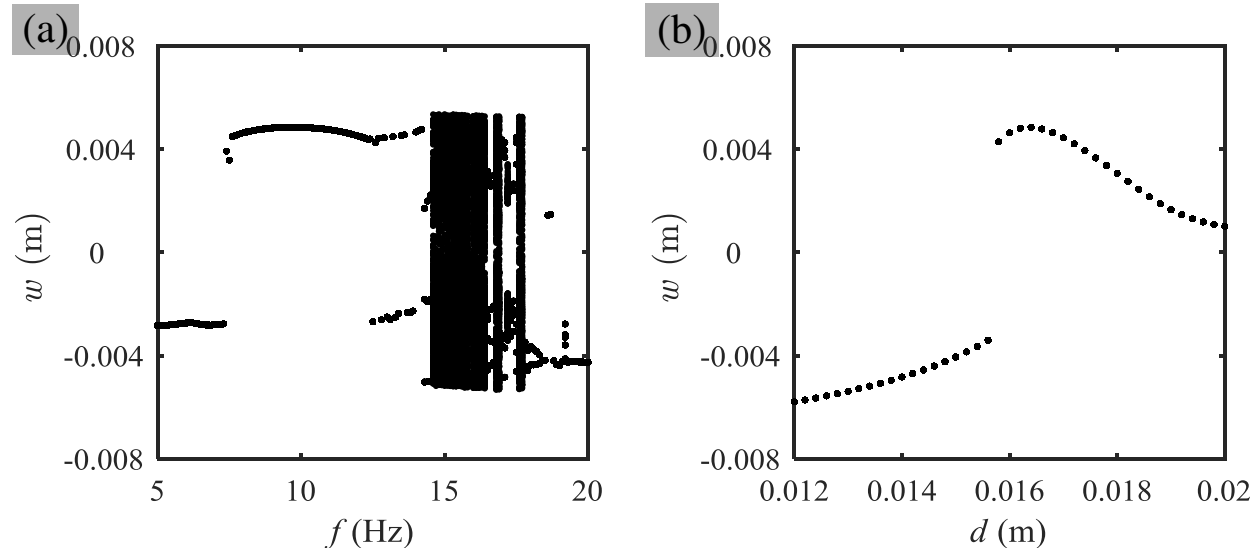

Fig. 12 Bifurcation diagrams of the friction plate's vibration amplitudes $w$ for (a) the excitation frequency $f$ when the acceleration excitation amplitude $A=1 \mathrm{~g}$, and the horizontal distance $d=0.016$ m, (b) the horizontal distance $\boldsymbol{d}$ between magnets with the excitation amplitude $A=1 \mathrm{~g}$, and the excitation frequency $f=8 \mathrm{~Hz}$.

Fig. 13 shows the responses of the TEH at an acceleration excitation amplitude of $A=1 \mathrm{~g}$ and the horizontal distance $d=0.016 \mathrm{~m}$ between the tip and fixed magnets. As shown in Fig. 13a, the TEH cannot cross the potential barrier induced by the magnet pairs, resulting in a periodic intrawell oscillations around one of the stable equilibria. The response amplitudes are small and the efficiency of harvesting the vibration energy is low. With the increase of the excitation frequency, the TEH crosses the potential energy barrier, and the motion pattern becomes interwell periodic 
oscillations, as illustrated in Fig. 13b. Compared with the intrawell oscillation, the interwell one generates larger response amplitude, which is beneficial for converting the mechanical energy from the ambient vibration. As the excitation frequency continues to increase, the TEH oscillates around one of the stable equilibrium again with small amplitude, as delineated in Fig. 13c. However, when the excitation frequency is at $16 \mathrm{~Hz}$, the motion pattern of system turns from periodic oscillations into aperiodic/chaotic interwell oscillations, as illustrated in Fig. 13d.
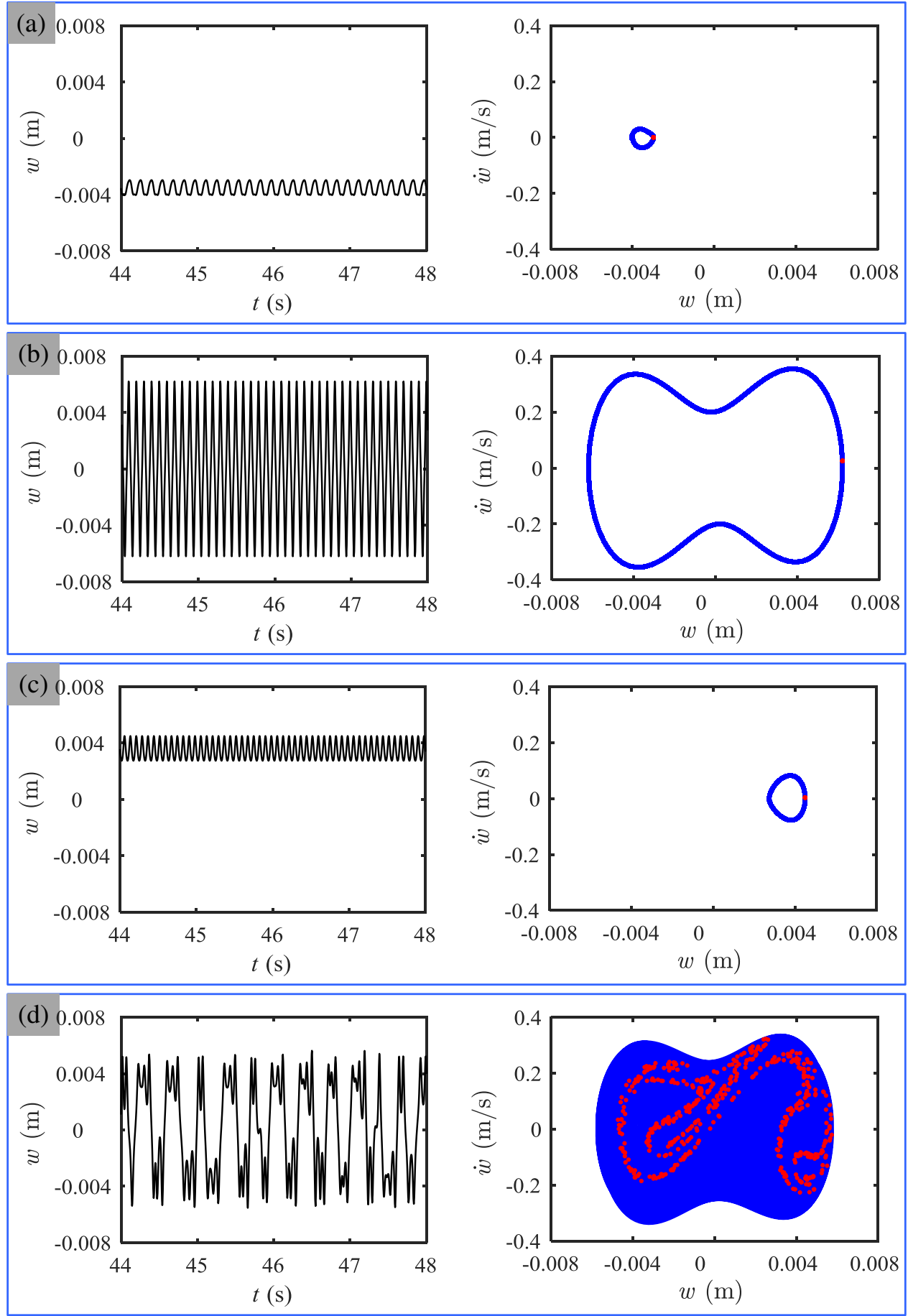
Fig. 13 Responses of the TEH at an acceleration excitation amplitude of $A=1 \mathrm{~g}$, and the horizontal distance $d=0.016 \mathrm{~m}$ and an excitation frequency of (a) $f=7 \mathrm{~Hz}$, (b) $f=10 \mathrm{~Hz}$, (c) $f=13 \mathrm{~Hz}$, (d) $f=16$ Hz.

In this energy harvesting system, the performance is strongly influenced by the horizontal distance between the tip magnet and the fixed magnet. Fig. 14 depicts the responses of the TEH for an excitation amplitude of $A=1 \mathrm{~g}$ and the excitation frequency of $f=10 \mathrm{~Hz}$. In all cases, the motion pattern of the TEH is periodic. When the horizontal distance is small, for example, $d=0.014 \mathrm{~m}$, the TEH undergoes intrawell oscillations, as shown in Fig. 14a. For such a motion pattern, the tip magnet cannot cross the potential barrier induced by the strong magnetic force. For a horizontal distance of $0.016 \mathrm{~m}$ (Fig. 14b), the TEH oscillates between the two equilibrium points periodically. As the increase in the horizontal distance from $0.018 \mathrm{~m}$ to $0.02 \mathrm{~m}$, the TEH keeps intrawell oscillations with gradually decreased amplitude. Indeed, such a variation trend of the motion pattern can be attributed to the change from bistablity to monostability (as reported in Fig. 6). 

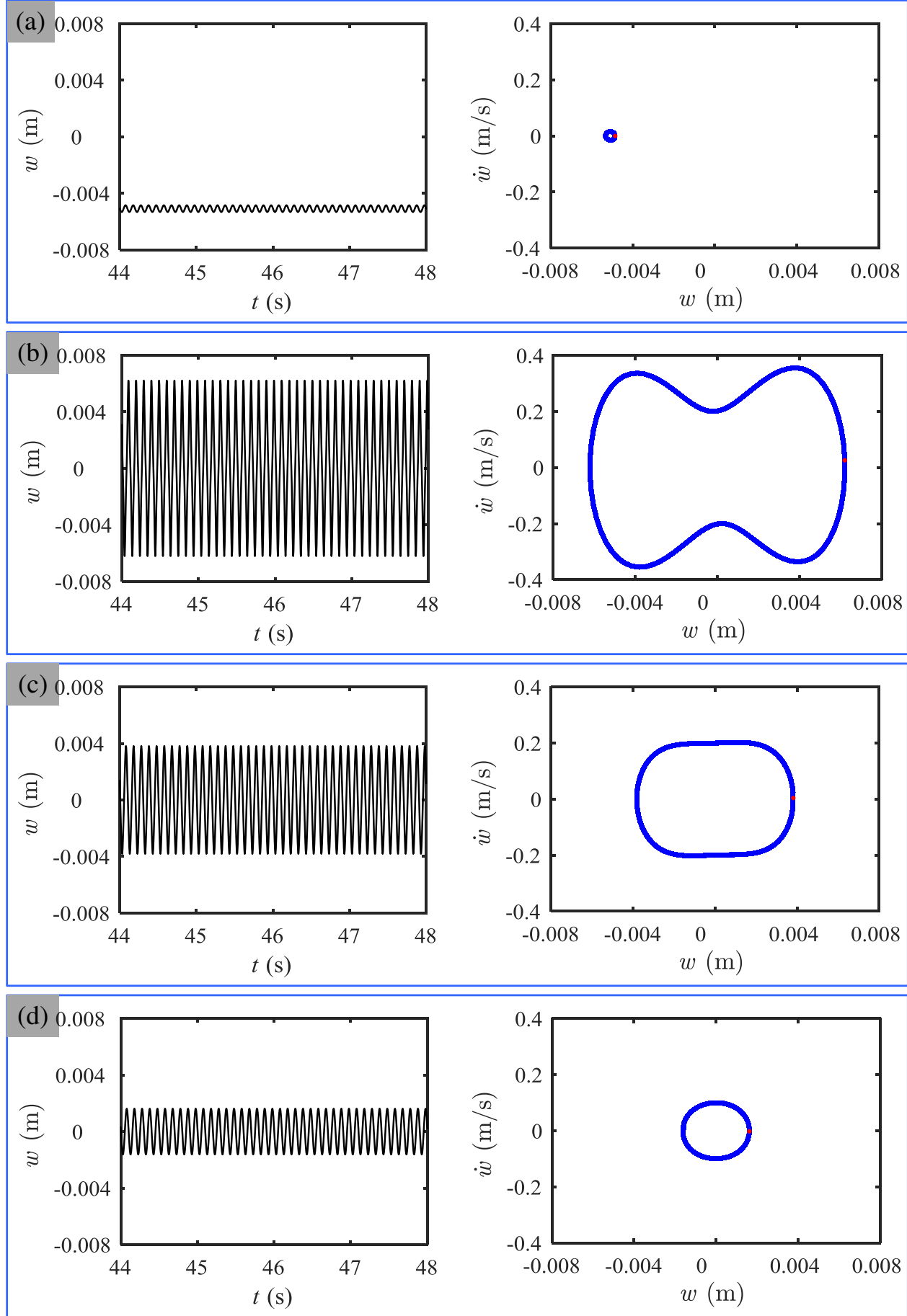

Fig. 14 The effect of the horizontal distance on the motion pattern when the excitation amplitude $A=1 \mathrm{~g}$, and the excitation frequency of $f=10 \mathrm{~Hz}$. (a) $d=0.014 \mathrm{~m}$, (b) $d=0.016 \mathrm{~m}$, (c) $d=0.018 \mathrm{~m}$, (d) $d=0.02 \mathrm{~m}$.

Fig. 15 shows the bifurcation diagrams of the vibration amplitudes with respect to the normal force for different excitation frequencies when the acceleration excitation amplitude is $A=1 \mathrm{~g}$ and the horizontal distance is $d=0.016 \mathrm{~m}$. As shown in 
Fig. 15a, the motion pattern of TEH is periodic in the whole range of the normal force. As the excitation frequency increases (such as $f=14 \mathrm{~Hz}$ ), the periodic motion (when the normal force is small) coexists with the aperiodic/chaotic oscillations (when the normal force is large), as presented in Fig. 15b. At excitation frequency of $17 \mathrm{~Hz}$, the oscillations of the system are aperiodic/chaotic, as seen in Fig.15c. In the end, the vibrations of the TEH become periodic as long as the excitation frequency is sufficiently large, as illustrated in Fig. 15d.
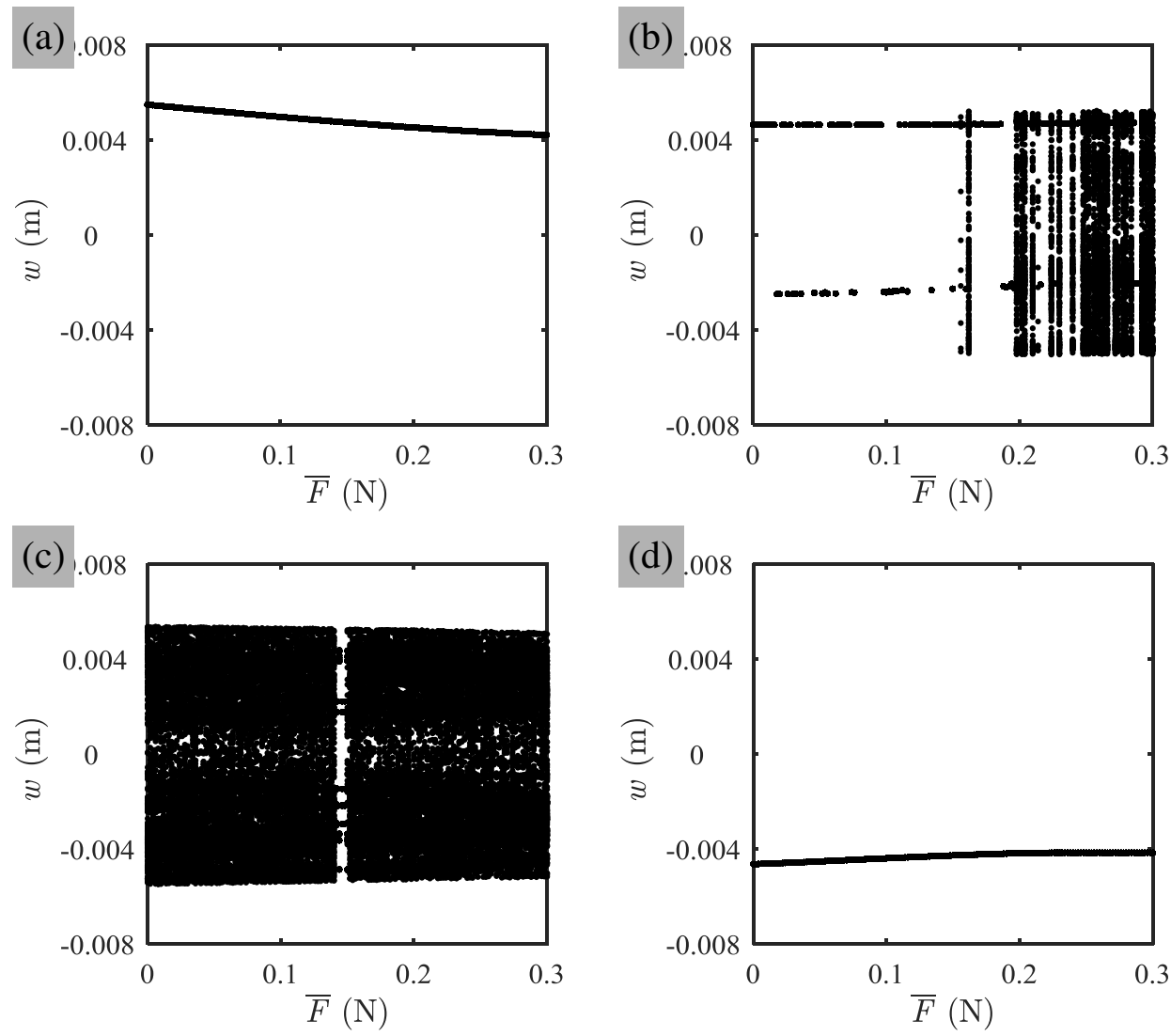

Fig. 15 Bifurcation diagrams of the vibration amplitudes $w$ for the normal force $F_{\mathrm{N}}$ when the excitation frequency of (a) $f=11 \mathrm{~Hz}$, (b) $f=14 \mathrm{~Hz}$, (c) $f=17 \mathrm{~Hz}$, (d) $f=20 \mathrm{~Hz} ; A=1.0 \mathrm{~g}, d=0.016 \mathrm{~m}$

\section{Electrical performance of the TEH}

In the overlapped region of Dielectric 1 and Dielectric 2, the electric field $E_{1}$ and $E_{2}$ can be written as [9] 


$$
\begin{aligned}
& E_{1}=\frac{\sigma x}{\varepsilon_{0} \varepsilon_{r 1}(l-x)} \\
& E_{2}=\frac{\sigma x}{\varepsilon_{0} \varepsilon_{r 2}(l-x)}
\end{aligned}
$$

where $\sigma$ is the charge density of the Dielectric 1 and Dielectric 2 when they slide relative to each other, $l$ is the length of Dielectric 1 and Dielectric 2, and $x$ is the distance of separation between Dielectric 1 and Dielectric 2. $\varepsilon_{0}$ is the vacuum permittivity, and $\varepsilon_{\mathrm{r} 1}$ and $\varepsilon_{\mathrm{r} 2}$ denote the relative permittivity of Dielectric 1 and Dielectric 2, respectively.

The open-circuit voltage of the energy harvesting system can be given as

$$
V_{\mathrm{OC}}=E_{1} d_{1}+E_{2} d_{2}=\frac{\sigma x}{\varepsilon_{0}(l-x)}\left(\frac{d_{1}}{\varepsilon_{r 1}}+\frac{d_{2}}{\varepsilon_{r 2}}\right)
$$

where $d_{1}$ and $d_{2}$ are the thicknesses of Dielectric 1 and Dielectric 2, respectively.

The parameters utilized in electrical simulations are tabulated in Table 2.

Table 2 The parameters utilized in the electrical simulations

\begin{tabular}{ll}
\hline Parameter & Value \\
\hline Dielectric 1 & $\varepsilon_{r 1}=4, d_{1}=2.2 \times 10^{-4} \mathrm{~m}$ \\
Dielectric 2 & $\varepsilon_{r 2}=4, d_{2}=2.2 \times 10^{-4} \mathrm{~m}$ \\
Width of the dielectrics & $b=0.01 \mathrm{~m}$ \\
Length of the dielectrics & $l=0.01 \mathrm{~m}$ \\
Surface tribo-charge density & $\sigma=6 \mu \mathrm{C} / \mathrm{m}^{2}$ \\
Vacuum permittivity & $\varepsilon_{0}=8.85 \times 10^{-12} \mathrm{~F} / \mathrm{m}$ \\
\hline
\end{tabular}

Fig. 16 shows the numerical results of the open-circuit voltage of the TEH for different normal forces. When the normal force is tiny ( such as $F_{\mathrm{N}}=0.1 \mathrm{~N}$ ), the tip magnet can crosses the potential energy barrier, jumps out of the potential energy wells and undergoes large-amplitude interwell oscillations in low-frequency range, 
and thus the TEH exhibits high open-circuit voltage in low-frequency range. As the normal force increases (i.e., $F_{\mathrm{N}}=0.2 \mathrm{~N}$ and $F_{\mathrm{N}}=0.3 \mathrm{~N}$ ), both the amplitude and frequency bandwidth of open-circuit voltage decrease. The reason of this phenomenon is that both the equivalent stiffness and damping coefficient increase with the increase of the normal force. Thus, the impact of the normal force on energy harvesting by the sliding-mode TEH should be taken into account.

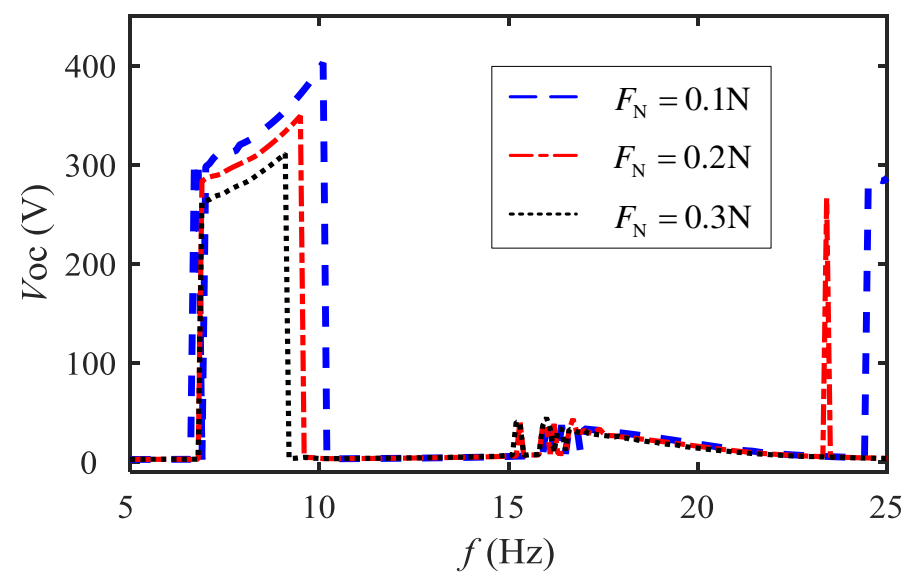

Fig. 16 Numerical results for the open-circuit voltage of the TEH under frequency sweeps with different normal forces by using Macro-slip friction model (blue) $F_{\mathrm{N}}=0.1 \mathrm{~N}$, (red) $F_{\mathrm{N}}=0.2 \mathrm{~N}$, (black) $F_{\mathrm{N}}=0.3 \mathrm{~N}, A=0.3 \mathrm{~g}$ and $d=0.016 \mathrm{~m}$.

As delineated in Fig. 17, an optimal horizontal distance between magnets is benefit to improve energy harvesting efficiency. When the distance $d=0.014 \mathrm{~m}$, no voltage is generated, because the motion pattern of TEH hardly changes in potential wells. As the distance increases (such as $d=0.016 \mathrm{~m}$ ), large-amplitude interwell oscillations appear, and thus high open-circuit voltage of the TEH exhibits in low-frequency range. With the increase in the horizontal distance from $0.016 \mathrm{~m}$ to $0.018 \mathrm{~m}$, the TEH turns from bistable into monostable, and the open-circuit voltage with low amplitude shows in the whole frequency range. 


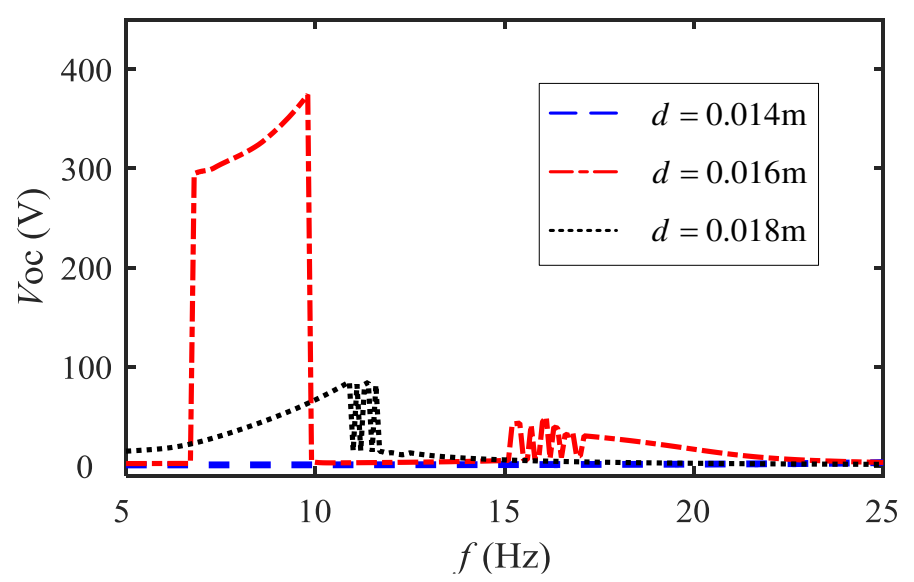

Fig. 17 The electrical responses of TEH under frequency sweeps with the different horizontal distances $d$ between the tip magnet and the fixed magnet (blue) $d=0.014 \mathrm{~m}$, (red) $d=0.016 \mathrm{~m}$, (black) $d=0.018 \mathrm{~m}, A=0.3 \mathrm{~g}$ and $F_{\mathrm{N}}=0.15 \mathrm{~N}$.

Fig. 18 shows the relationship between the open-circuit voltage and the frequency for different acceleration amplitudes of the base excitation. Clearly, when the acceleration amplitude is weak, the system oscillates slightly in one potential energy well. However, the tip magnet can cross the potential energy barrier in low-frequency range as the acceleration amplitude increases. Therefore, one can see from Fig. 18 that both the amplitude and frequency bandwidth of open-circuit voltage increase with the increase in the acceleration amplitude.

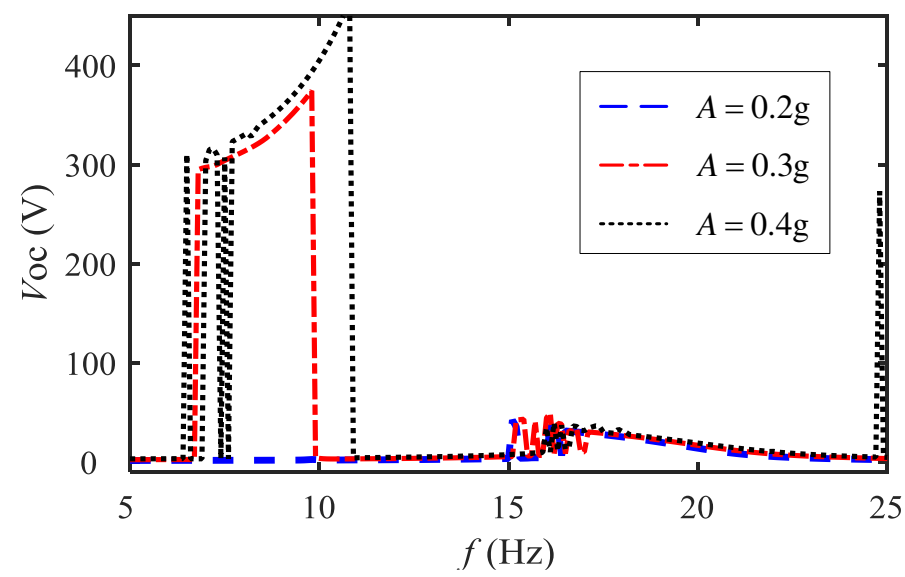

Fig. 18 The open-circuit voltage of the TEH under frequency sweeps with different acceleration amplitudes of the base excitation, (blue) $A=0.2 \mathrm{~g}$, (red) $A=0.3 \mathrm{~g}$, (black) $A=0.4 \mathrm{~g}, d=0.016 \mathrm{~m}$ and $F_{\mathrm{N}}=0.15 \mathrm{~N}$. 


\section{Conclusions}

This paper proposed a nonlinear sliding-mode TEH by equipping friction plate with a bird-shaped cantilever. Firstly, the conceptual design scheme of the nonlinear TEH is elaborated, and the dynamic model is established based on the Macro-slip friction theory. Then, the effects of the friction in sliding-mode TEH on the dynamic behaviors and electrical performances are studied in detail. The effects of parameters on both the mechanical and electrical responses are also discussed. The main conclusions are given as follows.

1. The Macro-slip friction model is equivalent to Coulomb friction model when the normal force is sufficient small and sufficient large. Nevertheless, the Coulomb friction model would be unable to predict the dynamic responses of the TEH, when the normal force is in a certain range, owing to neglecting the tangential contact stiffness.

2. With the increase of the normal force or the friction coefficient, the frequency band of the interwell oscillations decreases, leading to a performance degradation of energy harvesting.

3. When the magnets are close or far from each other, the system oscillates slightly in the potential energy wells. There exist a proper distance making the TEH oscillates between the potential energy wells to fulfill excellent performance of energy harvesting.

4. When the base excitation is tiny, the TEH cannot cross energy barrier and thus undergoes intrawell oscillations only. Interwell oscillations coexist with intrawell oscillations when the base excitation is sufficient large. With the increase in the base excitation, the frequency band of interwell oscillations becomes broader.

\section{Acknowledgments}

This research work was supported by National Natural Science Foundation of 
China (11972152, 12002122) and China Postdoctoral Science Foundation (2020M672476).

\section{Declarations of interest}

The authors declare no conflict of interest.

\section{References}

1. Thompson, J., Ge, X., Wu, H.C., Irmer, R., Jiang, H., Fettweis, G., Alamouti, S.: 5G wireless communication systems: Prospects and challenges [Guest Editorial]. IEEE Commun. Mag. 52, 62-64 (2014). https://doi.org/10.1109/MCOM.2014.6736744

2. Roh, W., Seol, J.-Y., Park, J., Lee, B., Lee, J., Kim, Y., Cho, J., Cheun, K., Samsung Electronics Co., L., Aryanfar, F.: Millimeter-wave beamforming as an enabling technology for $5 \mathrm{G}$ cellular communications: theoretical feasibility and prototype results. IEEE Commun. Mag. 52, 106-113 (2014). https://doi.org/10.1080/09513590.2017.1399696

3. Castagnetti, D.: A simply tunable electromagnetic pendulum energy harvester. Meccanica. 54, 749-760 (2019). https://doi.org/10.1007/s11012-019-00976-7

4. Halim, M.A., Rantz, R., Zhang, Q., Gu, L., Yang, K., Roundy, S.: An electromagnetic rotational energy harvester using sprung eccentric rotor, driven by pseudo-walking motion. Appl. Energy. 217, 66-74 (2018). https://doi.org/10.1016/j.apenergy.2018.02.093

5. Cao, J., Syta, A., Litak, G., Zhou, S., Inman, D.J., Chen, Y.: Regular and chaotic vibration in a piezoelectric energy harvester with fractional damping. Eur. Phys. J. Plus. 130, 103 (2015). https://doi.org/10.1140/epjp/i2015-15103-8

6. Litak, G., Friswell, M.I., Adhikari, S.: Regular and chaotic vibration in a piezoelectric energy harvester. Meccanica. 51, 1017-1025 (2016). https://doi.org/10.1007/s11012-015-0287-9

7. Yang, Y., Guo, W., Pradel, K.C., Zhu, G., Zhou, Y., Zhang, Y., Hu, Y., Lin, L., Wang, Z.L.: Pyroelectric nanogenerators for harvesting thermoelectric energy. Nano Lett. 12, 2833-2838 (2012). https://doi.org/10.1021/nl3003039

8. Xie, M., Zabek, D., Bowen, C., Abdelmageed, M., Arafa, M.: Wind-driven pyroelectric energy harvesting device. Smart Mater. Struct. 25, 125023 (2016). https://doi.org/10.1088/0964-1726/25/12/125023

9. $\quad$ Niu, S., Liu, Y., Wang, S., Lin, L., Zhou, Y.S., Hu, Y., Wang, Z.L.: Theory of sliding-mode triboelectric nanogenerators. Adv. Mater. 25, 6184-6193 (2013). https://doi.org/10.1002/adma.201302808

10. Qiu, G.L., Liu, W., Han, M. Di, Cheng, X.L., Meng, B., Smitha, A.S., Zhao, J.M., Zhang, H.X.: A cubic triboelectric generator as a self-powered orientation sensor. Sci. China Technol. Sci. 58, 842-847 (2015). https://doi.org/10.1007/s11431-015-5790-7

11. Zhou, Z., Qin, W., Zhu, P.: A broadband quad-stable energy harvester and its advantages over bi-stable harvester: Simulation and experiment verification. Mech. Syst. Signal Process. 84, 
158-168 (2017). https://doi.org/10.1016/j.ymssp.2016.07.001

12. Xue, H., Yang, Q., Wang, D., Luo, W., Wang, W., Lin, M., Liang, D., Luo, Q.: A wearable pyroelectric nanogenerator and self-powered breathing sensor. Nano Energy. 38, 147-154 (2017). https://doi.org/10.1016/j.nanoen.2017.05.056

13. Fan, F.R., Tian, Z.Q., Lin Wang, Z.: Flexible triboelectric generator. Nano Energy. 1, 328-334 (2012). https://doi.org/10.1016/j.nanoen.2012.01.004

14. Jin, C., Kia, D.S., Jones, M., Towfighian, S.: On the contact behavior of micro-/nano-structured interface used in vertical-contact-mode triboelectric nanogenerators. Nano Energy. 27, 68-77 (2016). https://doi.org/10.1016/j.nanoen.2016.06.049

15. Chandrasekhar, A., Alluri, N.R., Sudhakaran, M.S.P., Mok, Y.S., Kim, S.J.: A smart mobile pouch as a biomechanical energy harvester towards self-powered smart wireless power transfer applications. Nanoscale. 9, 9818-9824 (2017). https://doi.org/10.1039/c7nr00110j

16. Wang, S., Lin, L., Xie, Y., Jing, Q., Niu, S., Wang, Z.L.: Sliding-triboelectric nanogenerators based on in-plane charge-separation mechanism. Nano Lett. 13, 2226-2233 (2013). https://doi.org/10.1021/nl400738p

17. Yang, Y., Zhang, H., Chen, J., Jing, Q., Zhou, Y.S., Wen, X., Wang, Z.L.: Single-electrode-based sliding triboelectric nanogenerator for self-powered displacement vector sensor system. ACS Nano. 7, 7342-7351 (2013). https://doi.org/10.1021/nn403021m

18. He, C., Zhu, W., Gu, G.Q., Jiang, T., Xu, L., Chen, B.D., Han, C.B., Li, D., Wang, Z.L.: Integrative square-grid triboelectric nanogenerator as a vibrational energy harvester and impulsive force sensor. Nano Res. 11, 1157-1164 (2018).

https://doi.org/10.1007/s12274-017-1824-8

19. Bhatia, D., Kim, W., Lee, S., Kim, S.W., Choi, D.: Tandem triboelectric nanogenerators for optimally scavenging mechanical energy with broadband vibration frequencies. Nano Energy. 33, 515-521 (2017). https://doi.org/10.1016/j.nanoen.2017.01.059

20. Wu, C., Liu, R., Wang, J., Zi, Y., Lin, L., Wang, Z.L.: A spring-based resonance coupling for hugely enhancing the performance of triboelectric nanogenerators for harvesting low-frequency vibration energy. Nano Energy. 32, 287-293 (2017).

https://doi.org/10.1016/j.nanoen.2016.12.061

21. Wang, K., Zhou, J., Ouyang, H., Chang, Y., Xu, D.: A dual quasi-zero-stiffness sliding-mode triboelectric nanogenerator for harvesting ultralow-low frequency vibration energy. Mech. Syst. Signal Process. 151, 107368 (2021). https://doi.org/10.1016/j.ymssp.2020.107368

22. Lallart, M., Zhou, S., Yan, L., Yang, Z., Chen, Y.: Tailoring multistable vibrational energy harvesters for enhanced performance: theory and numerical investigation. Nonlinear Dyn. 96, 1283-1301 (2019). https://doi.org/10.1007/s11071-019-04853-6

23. Huang, D., Zhou, S., Litak, G.: Analytical analysis of the vibrational tristable energy harvester with a RL resonant circuit. Nonlinear Dyn. 97, 663-677 (2019). https://doi.org/10.1007/s11071-019-05005-6

24. Yuan, T., Yang, J., Chen, L.Q.: Nonlinear characteristic of a circular composite plate energy harvester: experiments and simulations. Nonlinear Dyn. 90, 2495-2506 (2017). https://doi.org/10.1007/s11071-017-3815-4

25. Ding, H., Chen, L.Q.: Designs, analysis, and applications of nonlinear energy sinks. Nonlinear Dyn. 100, 3061-3107 (2020). https://doi.org/10.1007/s11071-020-05724-1

26. Salauddin, M., Rasel, M.S., Kim, J.W., Park, J.Y.: Design and experiment of hybridized 
electromagnetic-triboelectric energy harvester using Halbach magnet array from handshaking vibration. Energy Convers. Manag. 153, 1-11 (2017). https://doi.org/10.1016/j.enconman.2017.09.057

27. Ibrahim, A., Ramini, A., Towfighian, S.: Triboelectric energy harvester with large bandwidth under harmonic and random excitations. Energy Reports. 6, 2490-2502 (2020). https://doi.org/10.1016/j.egyr.2020.09.007

28. Dhakar, L., Tay, F.E.H., Lee, C.: Development of a broadband triboelectric energy harvester with SU-8 micropillars. J. Microelectromechanical Syst. 24, 91-99 (2015). https://doi.org/10.1109/JMEMS.2014.2317718

29. Gupta, R.K., Shi, Q., Dhakar, L., Wang, T., Heng, C.H., Lee, C.: Broadband energy harvester using non-linear polymer spring and electromagnetic/triboelectric hybrid mechanism. Sci. Rep. 7, 1-13 (2017). https://doi.org/10.1038/srep41396

30. Zhao, C., Yang, Y., Upadrashta, D., Zhao, L.: Design, modeling and experimental validation of a low-frequency cantilever triboelectric energy harvester. Energy. 214, 118885 (2021). https://doi.org/10.1016/j.energy.2020.118885

31. Wang, K., Ouyang, H., Zhou, J., Chang, Y., Xu, D., Zhao, H.: A nonlinear hybrid energy harvester with high ultralow-frequency energy harvesting performance. Meccanica. (2021). https://doi.org/https://doi.org/10.1007/s11012-020-01291-2

32. Fu, Y., Ouyang, H., Davis, R.B.: Nonlinear dynamics and triboelectric energy harvesting from a three-degree-of-freedom vibro-impact oscillator. Nonlinear Dyn. 92, 1985-2004 (2018). https://doi.org/10.1007/s11071-018-4176-3

33. Yang, X., Wang, C., Lai, S.K.: A magnetic levitation-based tristable hybrid energy harvester for scavenging energy from low-frequency structural vibration. Eng. Struct. 221, 110789 (2020). https://doi.org/10.1016/j.engstruct.2020.110789

34. Wang, C., Lai, S.K., Wang, Z.C., Wang, J.M., Yang, W., Ni, Y.Q.: A low-frequency, broadband and tri-hybrid energy harvester with septuple-stable nonlinearity-enhanced mechanical frequency up-conversion mechanism for powering portable electronics. Nano Energy. 64, 103943 (2019). https://doi.org/10.1016/j.nanoen.2019.103943

35. Hartog, J.P.D.: Forced vibrations with combined viscous and coulomb damping. London, Edinburgh, Dublin Philos. Mag. J. Sci. 9, 801-817 (1930). https://doi.org/10.1080/14786443008565051

36. Menq, C.H., Griffin, J.H.: A comparison of transient and steady state finite element analyses of the forced response of a friotionaily damped beam. J. Vib. Acoust. Trans. ASME. 107, 19-25 (1985). https://doi.org/10.1115/1.3274709

37. Sanliturk, K.Y., Ewins, D.J.: Modelling two-dimensional friction contact and its application using Harmonic balance method. J. Sound Vib. 193, 511-523 (1996). https://doi.org/10.1006/jsvi.1996.0299

38. Asadi, K., Ahmadian, H., Jalali, H.: Micro/macro-slip damping in beams with frictional contact interface. J. Sound Vib. 331, 4704-4712 (2012). https://doi.org/10.1016/j.jsv.2012.05.026

39. Fu, Y., Ouyang, H., Benjamin Davis, R.: Nonlinear structural dynamics of a new sliding-mode triboelectric energy harvester with multistability. Nonlinear Dyn. 100, 1941-1962 (2020). https://doi.org/10.1007/s11071-020-05645-z

40. Yung, K.W., Landecker, P.B., Villani, D.D.: An Analytic Solution for the Force Between Two Magnetic Dipoles. Magn. Electr. Sep. 9, 39-52 (1998). https://doi.org/10.1155/1998/79537 
41. Stanton, S.C., McGehee, C.C., Mann, B.P.: Nonlinear dynamics for broadband energy harvesting: Investigation of a bistable piezoelectric inertial generator. Phys. D Nonlinear Phenom. 239, 640-653 (2010). https://doi.org/10.1016/j.physd.2010.01.019

42. Leng, Y.G., Gao, Y.J., Tan, D., Fan, S.B., Lai, Z.H.: An elastic-support model for enhanced bistable piezoelectric energy harvesting from random vibrations. J. Appl. Phys. 117, 064901 (2015). https://doi.org/10.1063/1.4907763

43. Li, D., Botto, D., Xu, C., Liu, T., Gola, M.: A micro-slip friction modeling approach and its application in underplatform damper kinematics. Int. J. Mech. Sci. 19, 105029 (2019). https://doi.org/10.1016/j.ijmecsci.2019.105029

44. Koh, K.-H., Griffin, J.H., Filippi, S., Akay, A.: Characterization of Turbine Blade Friction Dampers. J. Engi-neering Gas Turbines Power. 127, 237-245 (2005)

45. Olofsson, U., Hagman, L.: A model for micro-slip between flat surfaces based on deformation of ellipsoidal elastic bodies. Tribol. Int. 30, 599-603 (1997). https://doi.org/10.1016/S0301-679X(97)00028-5 
Figures

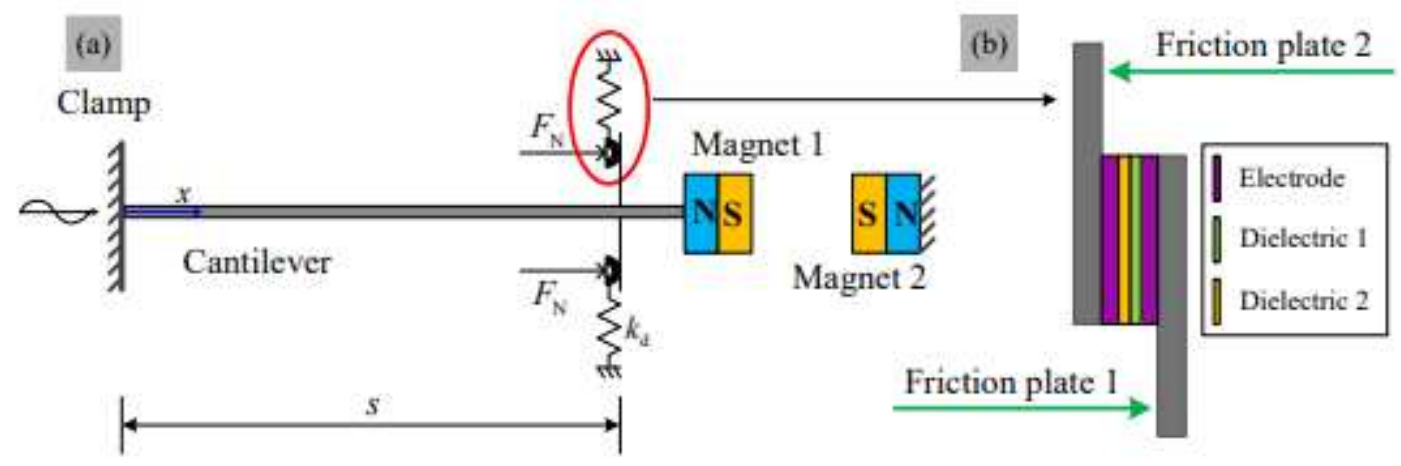

Figure 1

Conceptual model of the TEH

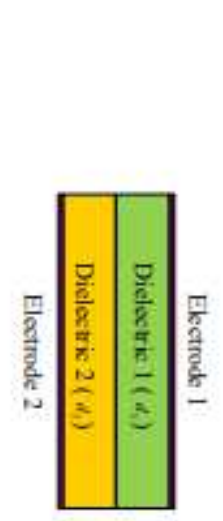

(a)

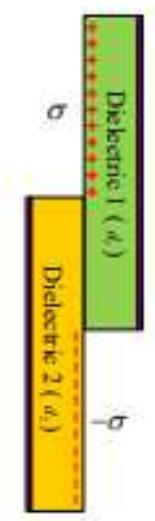

(b)

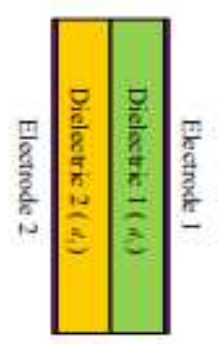

(c)

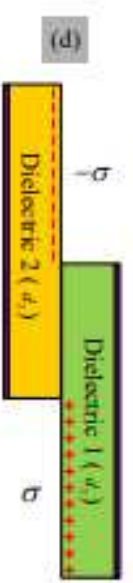

(c)

Figure 2

The operating principle of TEH 


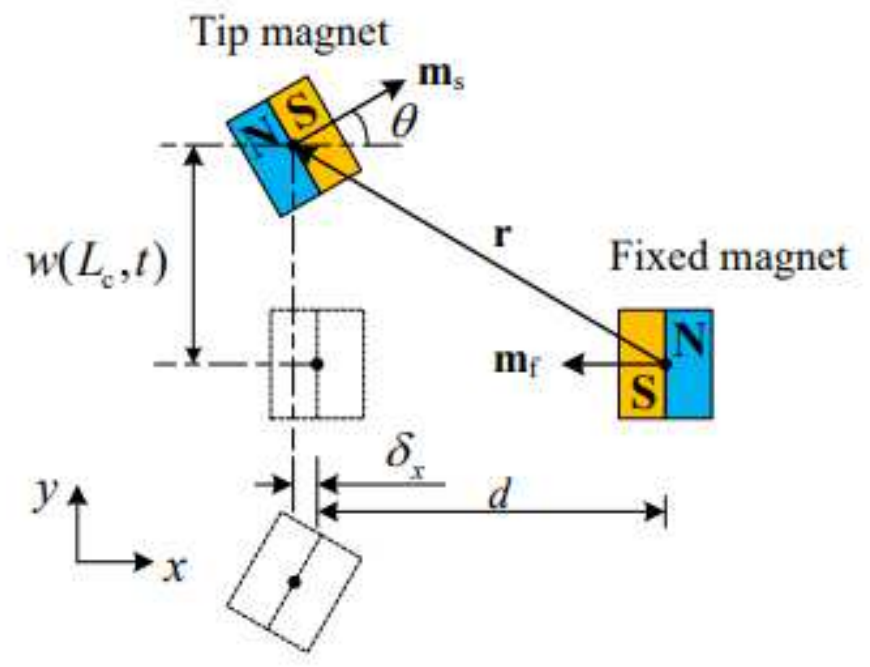

Figure 3

Geometries configuration of the two magnets

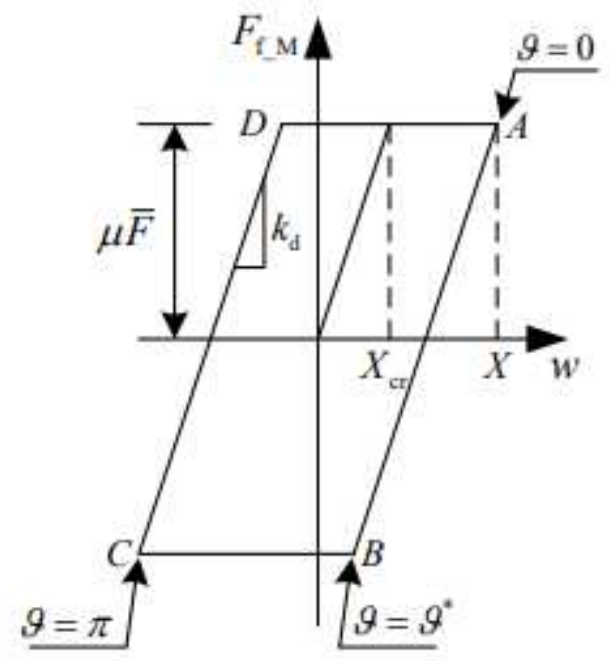

Figure 4

Frictional damping loop, friction force versus the response 

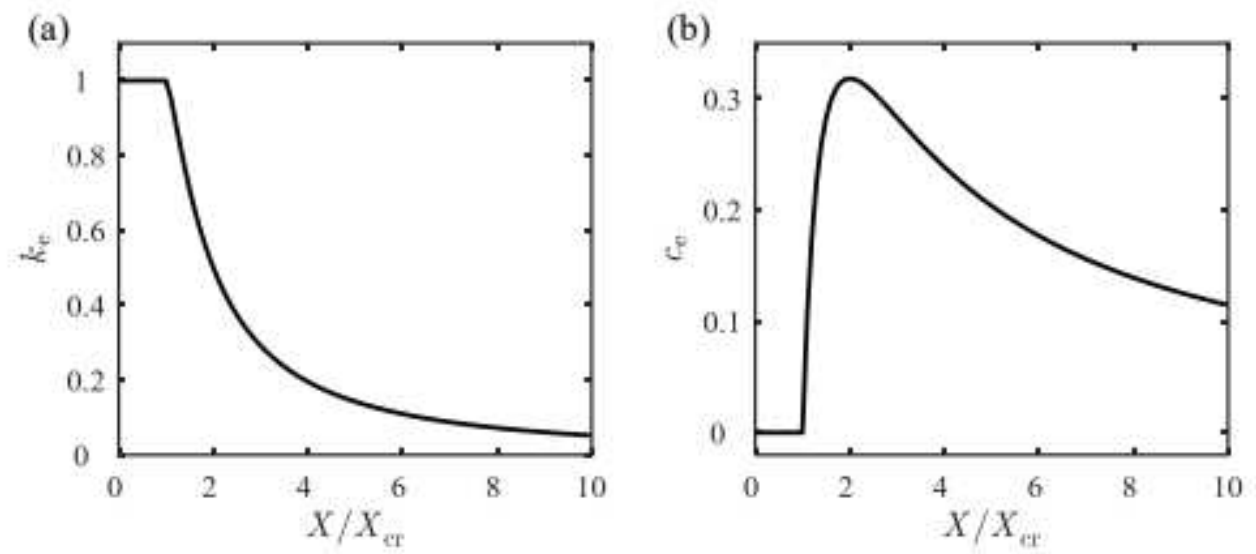

Figure 5

The variation of (a) equivalent stiffness, (b) equivalent damping coefficients
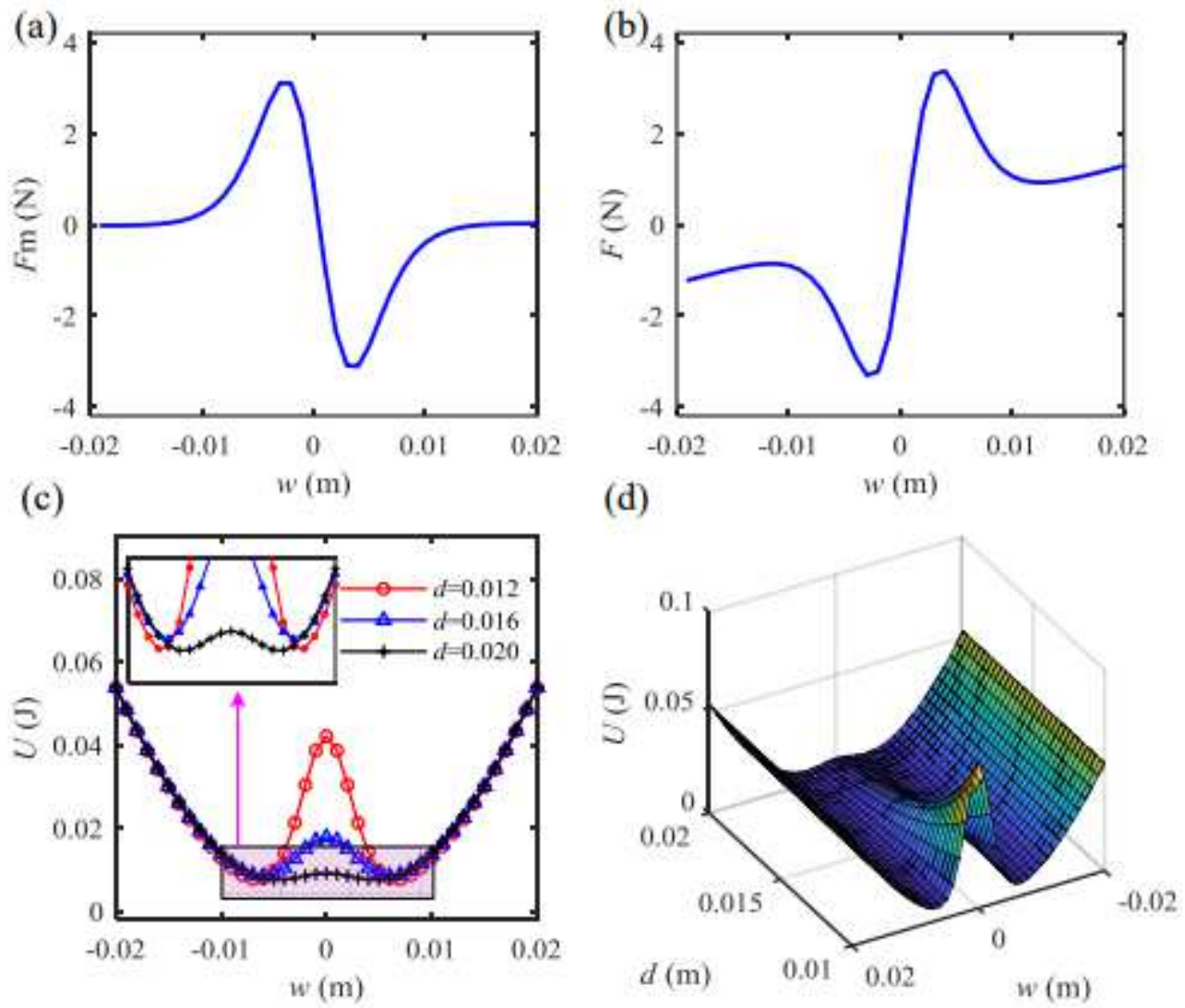

Figure 6

The variation of the force with the tip displacement, (a) the magnetic force, (b) the total force; the relationship between the total potential energy and the tip displacement, (c) for the different horizontal distances d, (d) a 3D surface plot under horizontal distances sweeps. 


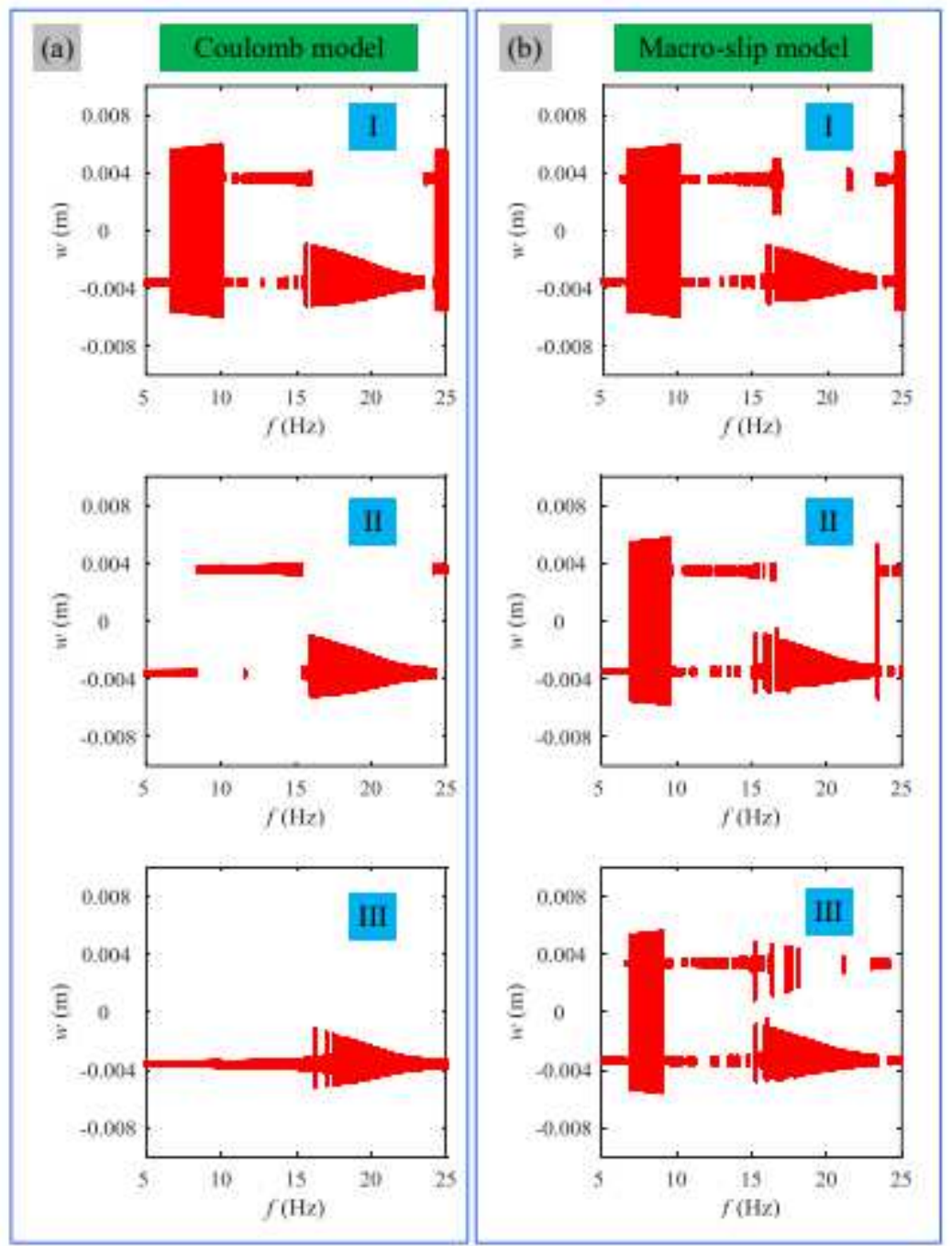

Figure 7

Please see the manuscript file to view the figure caption.

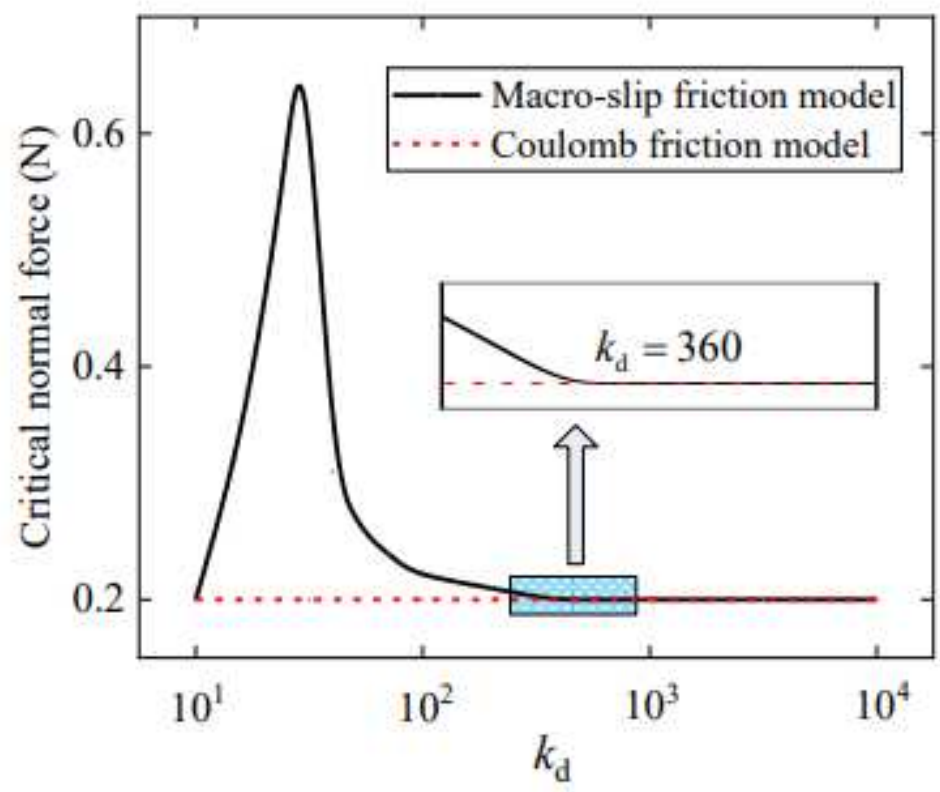


Figure 8

The critical normal force with different tangential contact stiffness

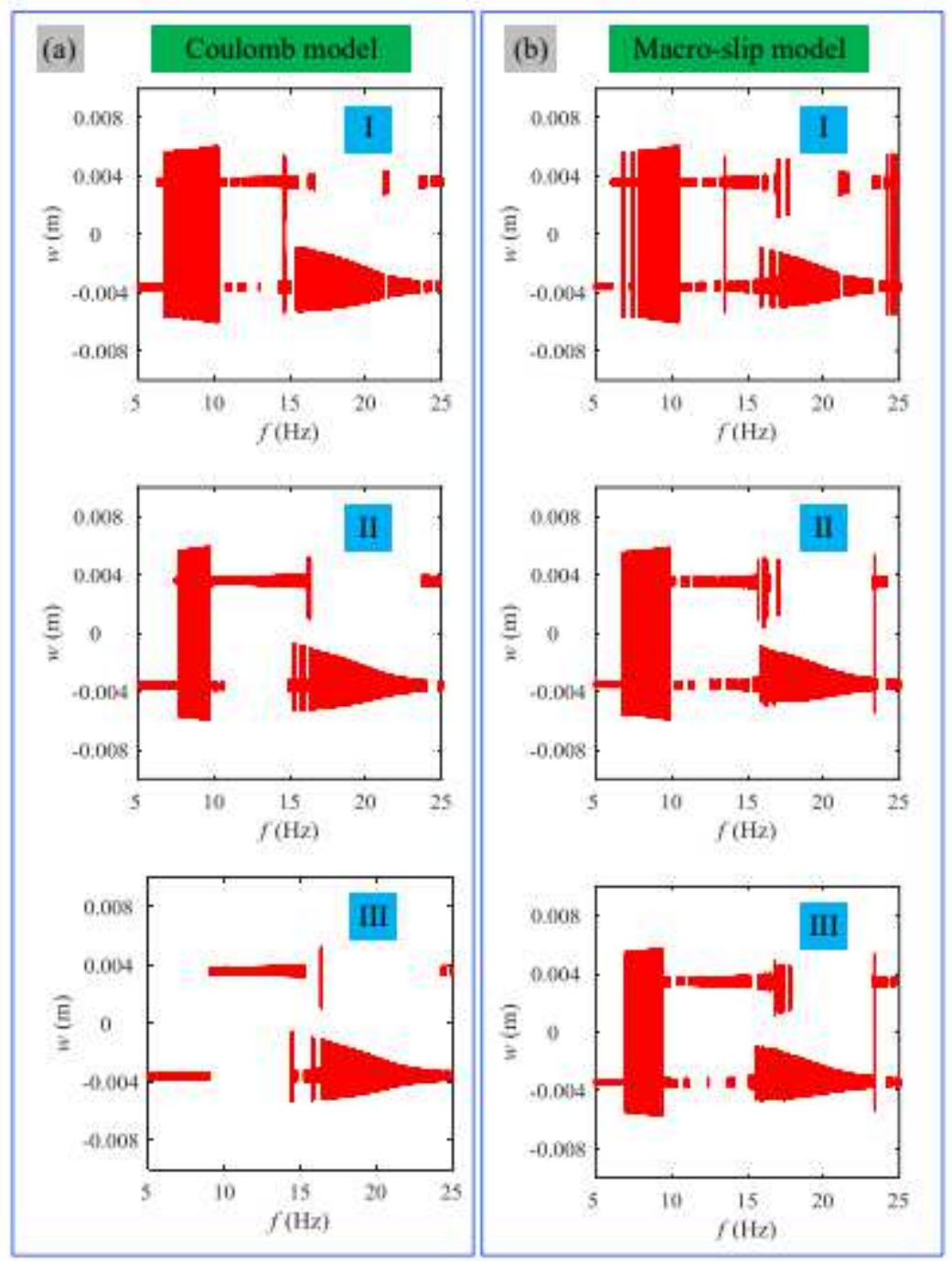

Figure 9

Please see the manuscript file to view the figure caption. 

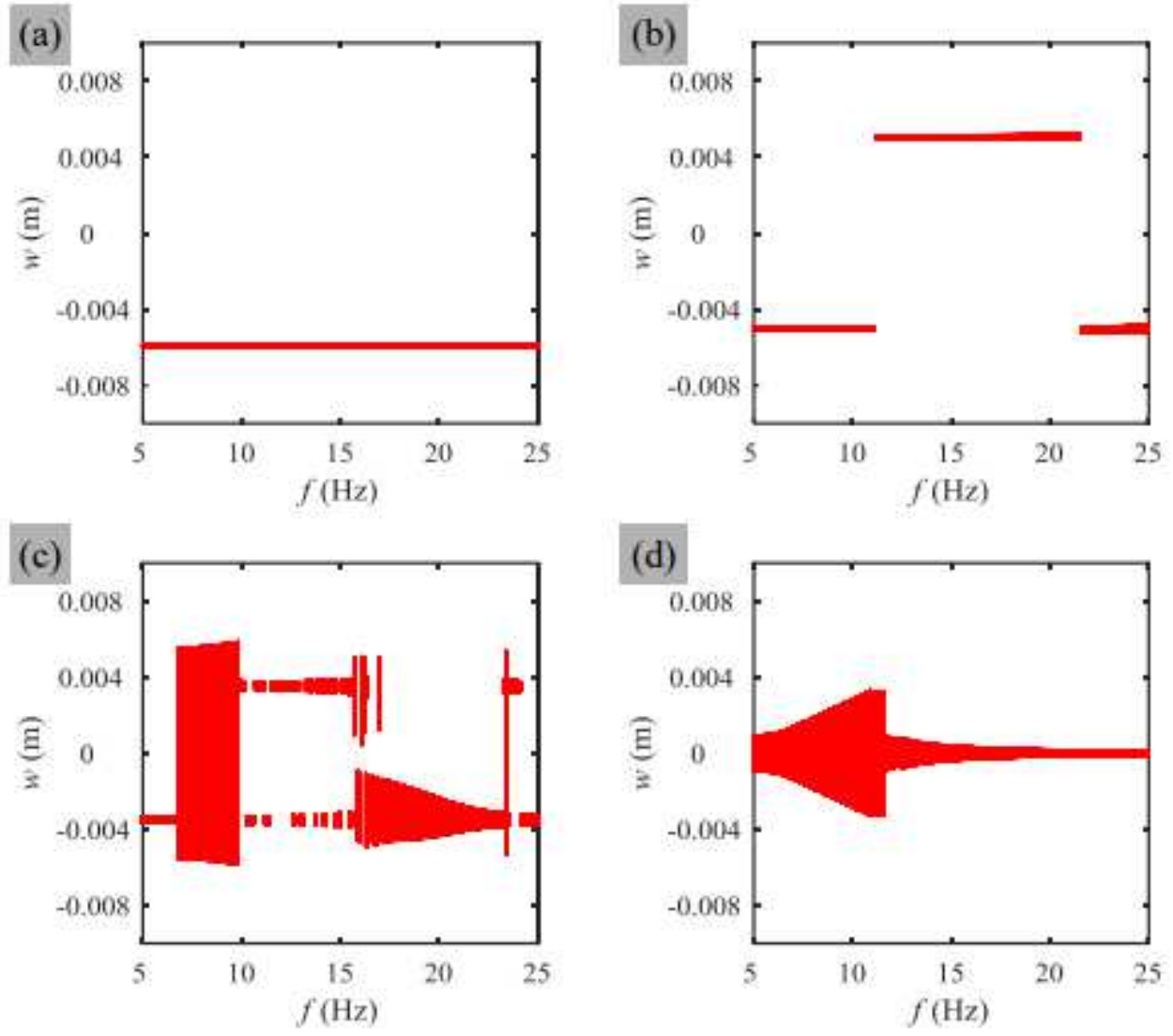

Figure 10

Please see the manuscript file to view the figure caption. 

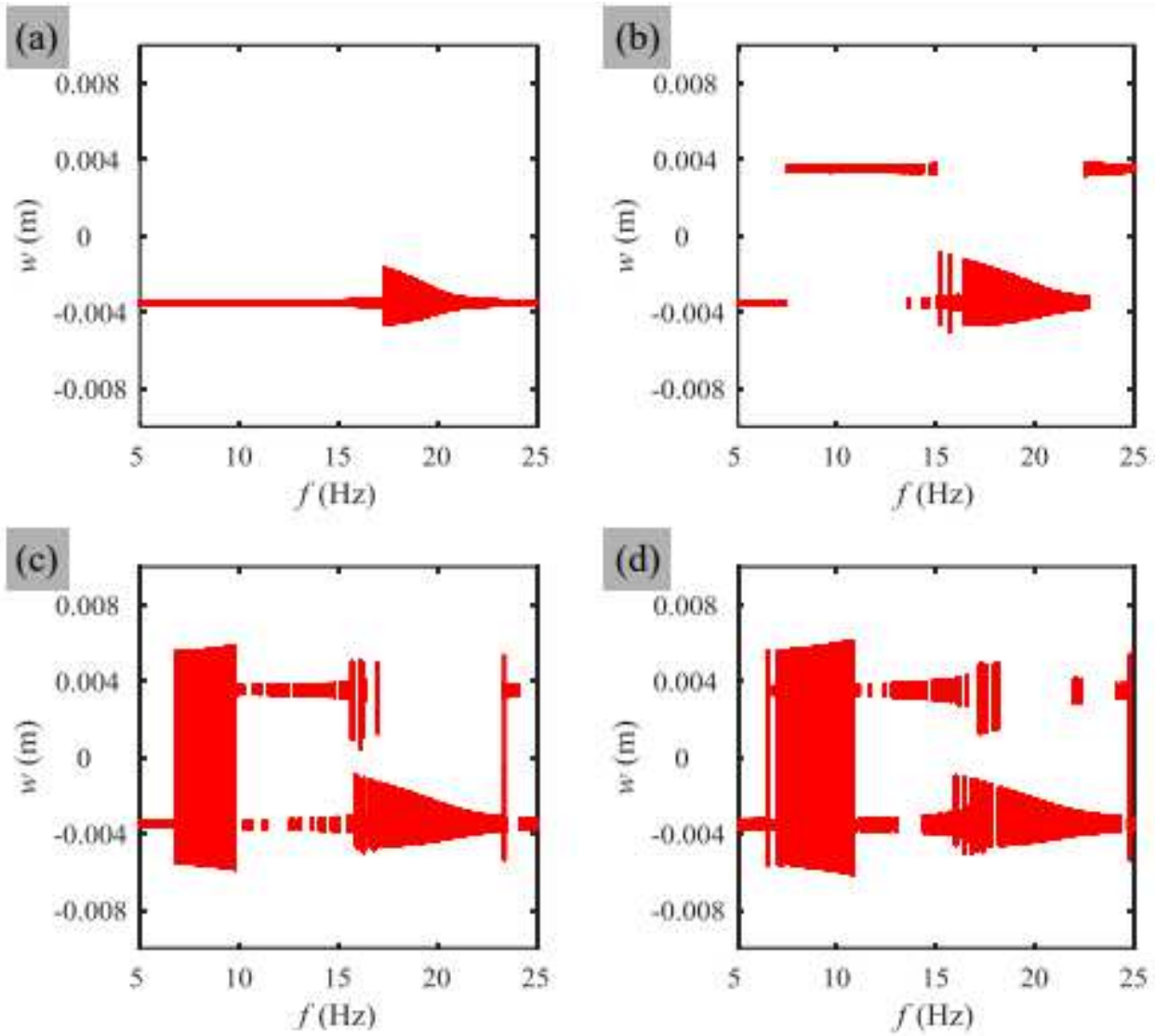

Figure 11

Please see the manuscript file to view the figure caption.
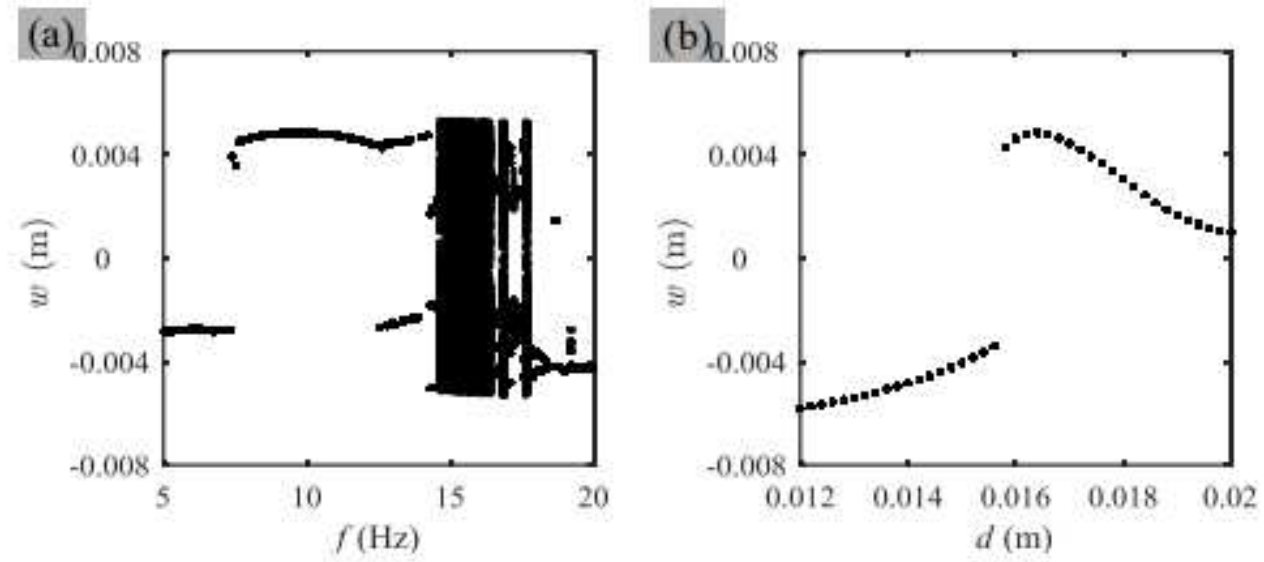

Figure 12

Bifurcation diagrams of the friction plate's vibration amplitudes $w$ for (a) the excitation frequency $f$ when the acceleration excitation amplitude $A=1 \mathrm{~g}$, and the horizontal distance $\mathrm{d}=0.016 \mathrm{~m}$, (b) the horizontal distance $d$ between magnets with the excitation amplitude $A=1 \mathrm{~g}$, and the excitation frequency $\mathrm{f}=8 \mathrm{~Hz}$. 

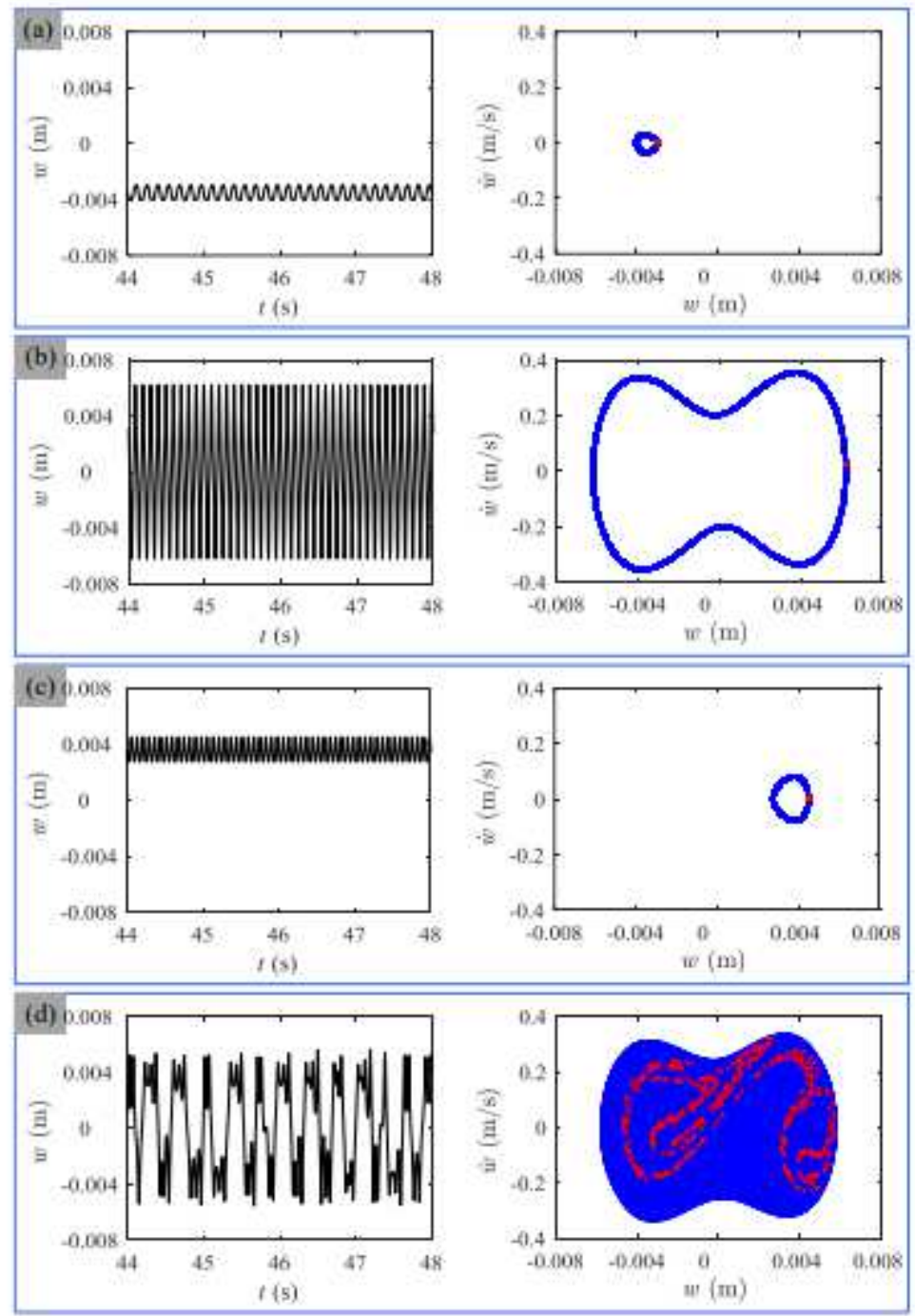

Figure 13

Responses of the TEH at an acceleration excitation amplitude of $A=1 \mathrm{~g}$, and the horizontal distance $d=0.016 \mathrm{~m}$ and an excitation frequency of (a) $f=7 \mathrm{~Hz}$, (b) $f=10 \mathrm{~Hz}$, (c) $f=13 \mathrm{~Hz}$, (d) $f=16 \mathrm{~Hz}$. 

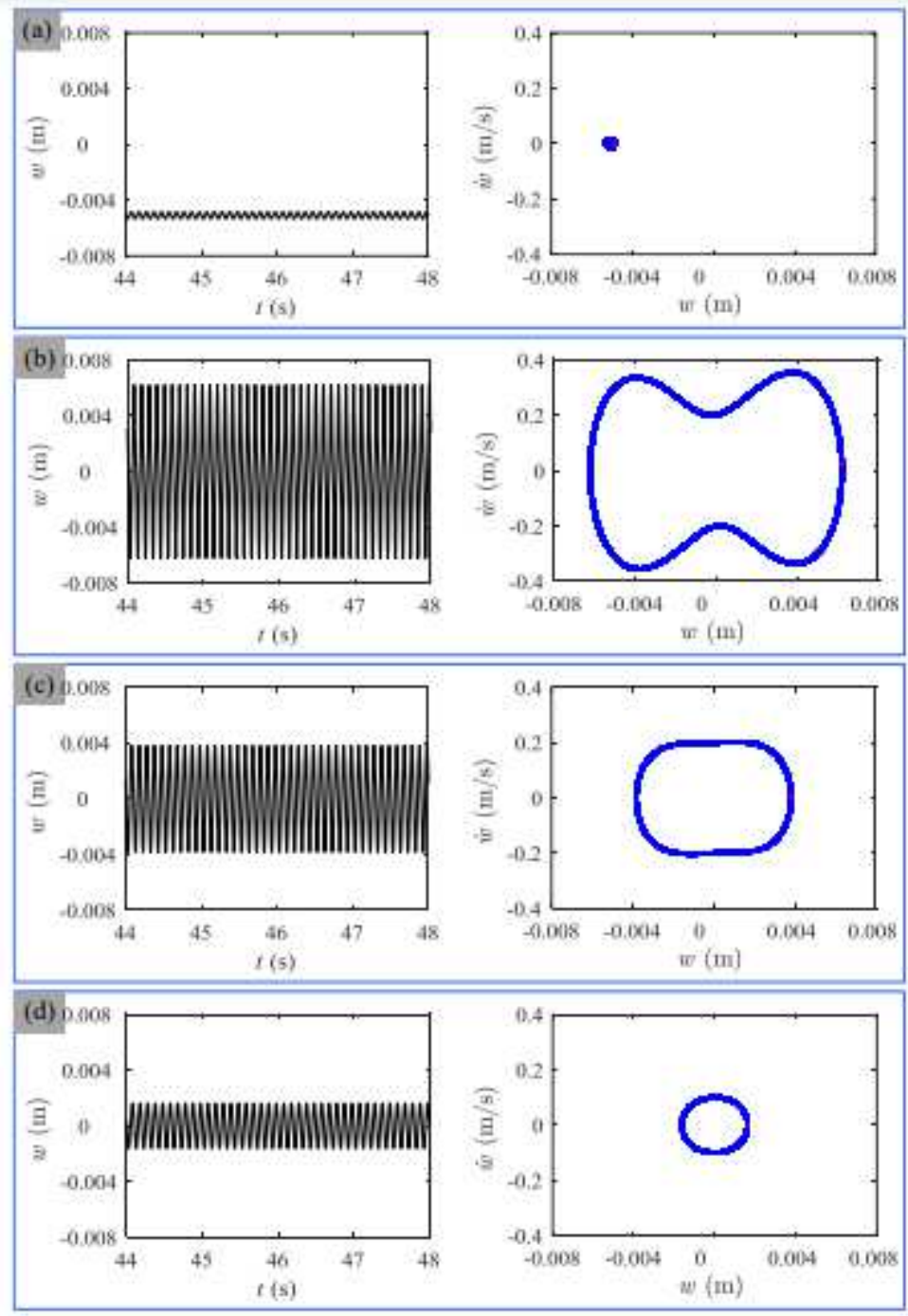

\section{Figure 14}

The effect of the horizontal distance on the motion pattern when the excitation amplitude $A=1 \mathrm{~g}$, and the excitation frequency of $f=10 \mathrm{~Hz}$. (a) $d=0.014 \mathrm{~m}$, (b) $d=0.016 \mathrm{~m}$, (c) $d=0.018 \mathrm{~m}$, (d) $d=0.02 \mathrm{~m}$. 

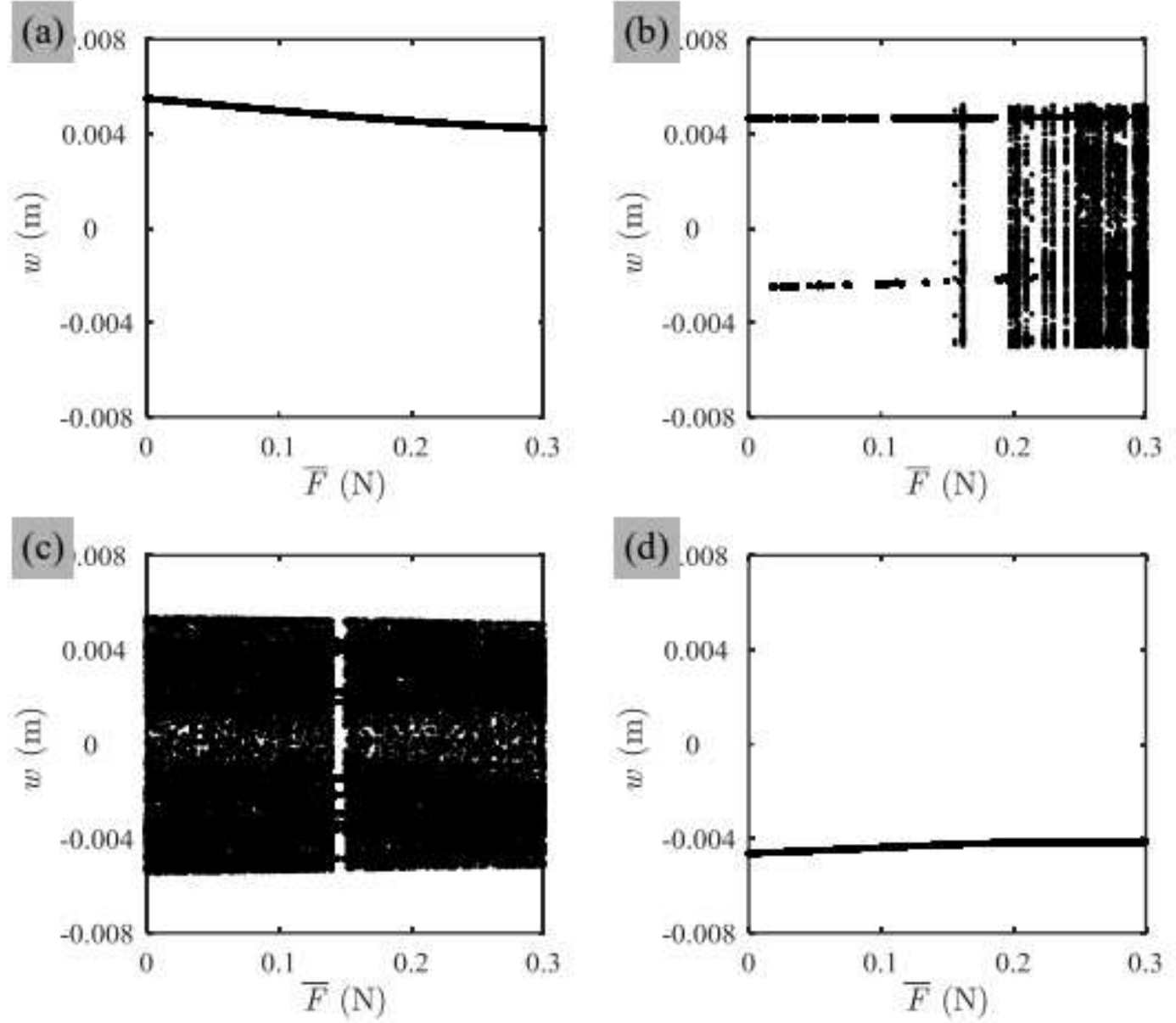

Figure 15

Bifurcation diagrams of the vibration amplitudes $w$ for the normal force $F N$ when the excitation frequency of (a) $f=11 \mathrm{~Hz}$, (b) $f=14 \mathrm{~Hz}$, (c) $f=17 \mathrm{~Hz}$, (d) $f=20 \mathrm{~Hz}$; $A=1.0 \mathrm{~g}, \mathrm{~d}=0.016 \mathrm{~m}$

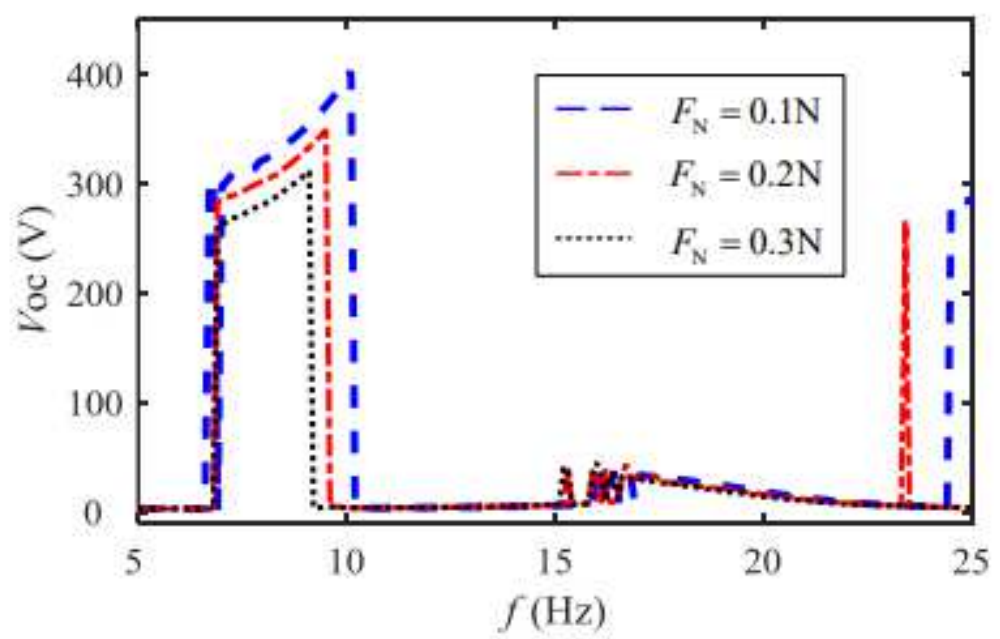

Figure 16

Please see the manuscript file to view the figure caption. 


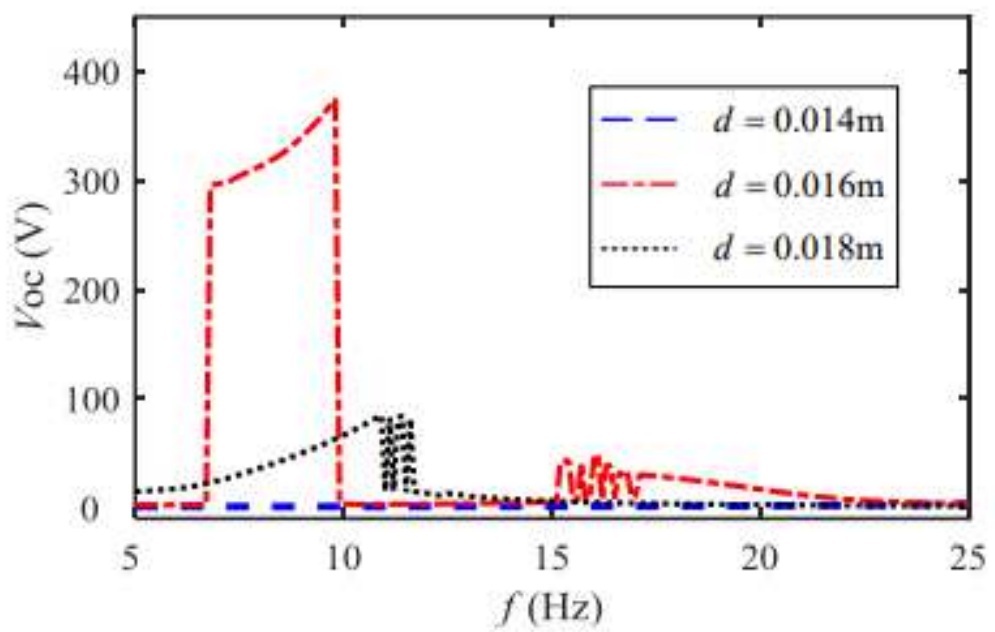

Figure 17

Please see the manuscript file to view the figure caption.

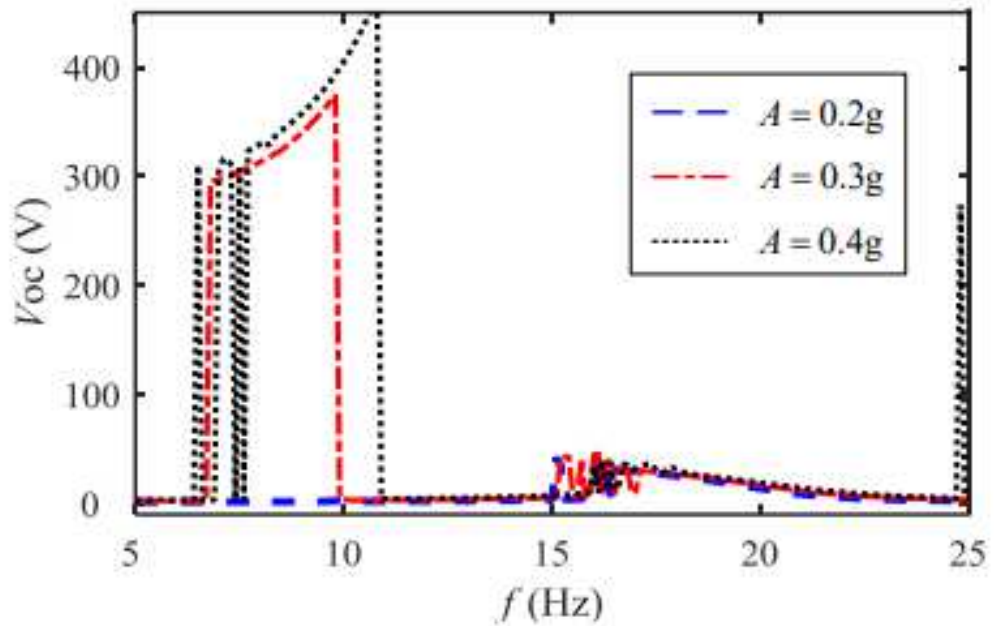

Figure 18

Please see the manuscript file to view the figure caption. 